\title{
Cross sections for meson-meson nonresonant reactions
}

\author{
Yu-Qi Li Xiao-Ming Xu \\ Department of Physics, Shanghai University, Baoshan, Shanghai 200444, China
}

\begin{abstract}
Meson-meson nonresonant reactions governed by the quark-interchange mechanism are studied in a potential that is derived from QCD. S-wave elastic phase shifts for $I=2 \pi \pi$ and $I=3 / 2 K \pi$ scattering are obtained with wave functions determined by the central spin-independent term of the potential. The reactions include inelastic scatterings of two mesons in the ground-state pseudoscalar octet and the ground-state vector nonet. Cross sections for reactions involving $\pi, \rho, K$ and $K^{*}$ indicate that mesonic interactions in matter consisting of only $K$ and $K^{*}$ can be stronger than mesonic interactions in matter consisting of only $\pi$ and $\rho$ and the reaction of $I=3 / 2 \pi K^{*} \rightarrow \rho K$ is most important among the endothermic nonresonant reactions. By the quark-interchange mechanism we can offer $\sqrt{s}$-dependences of $\phi$ absorption cross sections in collisions with $\pi$ and $\rho$ and relevant average cross sections what are very small for the reaction of $I=1 \pi \phi \rightarrow K^{*} K^{*}$ and remarkably large for the reaction of $I=1 \rho \phi \rightarrow K^{*} K^{*}$. It is found from the $\sqrt{s}$-dependences of cross sections that $\rho$ and $K^{*}$ creation cross sections can be larger than their absorption cross sections, respectively.
\end{abstract}

PACS: 25.75.-q; 13.75.Lb; 12.38.Mh

Keywords: Meson-meson nonresonant reactions; Cross sections; Quark-interchange mechanism. 


\section{Introduction}

Ratios of $p_{T}$-integrated mid-rapidity yields for mesons measured in PHENIX [1] for central $\mathrm{Au}+\mathrm{Au}$ collisions at $\sqrt{s_{N N}}=200 \mathrm{GeV}$ are $\pi^{-} / \pi^{+}=0.984, K^{-} / K^{+}=0.933$, $K^{+} / \pi^{+}=0.171$ and $K^{-} / \pi^{-}=0.162$, which agree with the measurements of other collaborations [2-5]. The ratio $\rho^{0} / \pi^{-}=0.169$ is obtained by the STAR Collaboration [6] in peripheral $\mathrm{Au}+\mathrm{Au}$ collisions at $\sqrt{s_{N N}}=200 \mathrm{GeV}$. The ratios $\pi^{-} / \pi^{+}$and $K^{-} / K^{+}$keep unchanged at $p_{T} \leq 2 \mathrm{GeV} / c$ or over the whole centrality range but $K^{+} / \pi^{+}$and $K^{-} / \pi^{-}$ increase with $p_{T}$ or centrality [1]. The ratios $K^{-} / \pi^{-}$and $K^{-} / K^{+}$do not vary near midrapidity and decrease slowly in the other measured rapidity region while both $\pi^{-} / \pi^{+}$and $K^{+} / \pi^{+}$are constants at $-3<y<3[2,7]$. It is evident from the above experimental results that pions, kaons, rhos are dominant meson species in hadronic matter. Subsequently, extended attention must be paid from the reactions $\pi \pi \rightarrow \pi \pi$ and $\pi \pi \rightarrow K \bar{K}$ in hadronic physics to a great number of other meson-meson reactions occurred in hadronic matter. On one hand meson-meson cross sections are crucial to chemical equilibration, thermalization, hadron flows and hadron yields, on the other hand earlier decoupled mesons due to small cross sections can show relatively clear information on quark-gluon plasma. Therefore, the study of meson-meson scatterings is a fundamental task in RHIC heavy-ion collisions.

A typical study of elastic $\pi \pi$ scattering is offered in chiral perturbation theory since pions represent Goldstone bosons. Phase shift for the elastic scattering at low energy can be well accounted for up to two loops that receive contributions from $\mathcal{L}_{2}, \mathcal{L}_{4}$ and $\mathcal{L}_{6}$ of the chiral Lagrangian for pion fields while the $\pi \pi$ scattering amplitude is constrained by analyticity, unitarity, crossing symmetry and chiral symmetry [8]. A combined study of the $I=0 \pi \pi \rightarrow \pi \pi$ and $\pi \pi \rightarrow K \bar{K}$ with Lippmann-Schwinger equations in coupled channels could also reproduce the phase shifts and inelasticities in the lowest order chiral Lagrangian that includes the pseudoscalar octet [9].

In order to explain the measured elastic phase shifts [10-15] for $I=2 \pi \pi$ and $I=$ $3 / 2 K \pi$ scattering, Barnes et al. $[16,17]$ considered Born-order quark-interchange diagrams in a nonrelativistic potential model and few other attempts have been made $[18,19]$. Motivated by the important $J / \psi$ suppression problem, the quark-interchange model in its reasonable form has been applied to study hadron- $J / \psi$ dissociation cross sections [20,21]. To the current need of obtaining unknown cross sections for meson-meson reactions in hadronic matter, the quark-interchange mechanism with a potential derived from QCD will at the present work produce new and useful results that include cross sections for the nonresonant reactions of $I=2 \pi \pi \leftrightarrow \rho \rho, I=2 \pi \rho \leftrightarrow \rho \rho, I=1 K K \leftrightarrow K^{*} K^{*}$, 
$I=1 K K^{*} \leftrightarrow K^{*} K^{*}, I=\frac{3}{2} \pi K \leftrightarrow \rho K^{*}, I=\frac{3}{2} \pi K^{*} \leftrightarrow \rho K^{*}, I=\frac{3}{2} \rho K \leftrightarrow \rho K^{*}$ and

$I=\frac{3}{2} \pi K^{*} \leftrightarrow \rho K$. These cross sections are unknown in both experiment and theory. The reactions originate from the quark-interchange process.

The $\phi$ mesons relevant to strangeness enhancement are used to conclude that the particle-identified elliptic flow coefficients have the scaling behavior over the constituentquark number [22-24]. Results of the $\phi$ meson measurements [25] are usually explained with the assumption that hadron- $\phi$ cross sections are so small that $\phi$ mesons will retain information from the early hot and dense matter [26-28]. Accordingly, it is very interesting to examine the assumption in theory. Meson-phi interactions may lead to collisional broadening of the phi meson width in spite of the small meson-phi cross sections that were obtained from effective meson Lagrangians [29-31]. The Lagrangians also give $\phi$ mean free path larger than that obtained from large collision rates of phi meson with $\rho, K$ and $K^{*}$ mesons as a result of the hidden local symmetry Lagrangian [32]. The discrepancy created from the two Lagrangians is due to the different evaluations of hadron-phi scatterings. To help understand the behavior of $\phi$ meson in hadronic matter, the hadron-phi cross sections need to be studied in different ways, especially in the attempt of quark level, which is just the second purpose of the present work. We calculate cross sections for the nonresonant reactions of $I=1 \pi \phi \rightarrow K K^{*}\left(\right.$ or $\left.K^{*} K\right), I=1 \pi \phi \rightarrow K^{*} K^{*}, I=1 \rho \phi \rightarrow K K$, $I=1 \rho \phi \rightarrow K K^{*}\left(\right.$ or $\left.K^{*} K\right)$ and $I=1 \rho \phi \rightarrow K^{*} K^{*}$. These cross sections have not been evaluated in quark models. The reactions arise out of the quark-interchange process.

In next section we describe the potential derived from QCD and mesonic quarkantiquark wave functions. Formulas established with the quark-interchange mechanism are presented in Section 3. Elastic phase shifts for $I=2 \pi \pi$ and $I=3 / 2 K \pi$ scattering, cross sections for the nonresonant reactions and discussions are given in Section 4 . Conclusions are in the last section.

\section{Potential and radial wave functions}

The Buchmüller-Tye potential [33] is a QCD-like potential because the running coupling constant is obtained from the $\beta$ function that takes a linear form in the strongcoupling limit is renormalization-scheme-independent and gauge-invariant up to two loops in the weak-coupling region and contains a term related to the three-loop contribution. The potential was nonrelativistic, central and spin-independent. However, the running coupling constant leads to a relativistic potential that takes as the first term the linear confinement and as the second term a relativistic one-gluon-exchange potential with the perturbative one- and two- loop corrections. In the second term the part responsible for 
the confinement is subtracted from the running coupling constant. We peform nine times of different Foldy-Wouthuysen canonical transformations to the two-constituent Hamiltonian with the relativistic potential. A lengthy calculation gives a central spin-independent term, spin-spin interaction, spin-orbit and tensor terms [34]. In this way the BuchmüllerTye potential is extended. The central spin-independent term, i.e. the Buchmüller-Tye potential, contains the linear confinement and loop corrections to the Coulomb potential. The term does not rely on the masses of quark and antiquark constituents. The spin-spin interaction includes a contact term and a loop-correction term. The spin-orbit and tensor terms contain factors that are given by loop corrections. The spin-spin, spin-orbit and tensor terms are related to the inverse of constituent mass. The spin-orbit and tensor terms do not contribute to the masses of mesons in the ground-state pseudoscalar octet and the ground-state vector nonet. The central spin-independent term is

$$
V_{\mathrm{si}}=-\frac{\vec{\lambda}_{a}}{2} \cdot \frac{\vec{\lambda}_{b}}{2} \frac{3}{4} \mathrm{k} r+\frac{\vec{\lambda}_{a}}{2} \cdot \frac{\vec{\lambda}_{b}}{2} \frac{6 \pi}{25} \frac{v(\lambda r)}{r}
$$

where $\vec{\lambda}_{a}$ and $\vec{\lambda}_{b}$ are the Gell-Mann " $\lambda$-matrices", $\mathrm{k}=1 / 2 \pi \alpha^{\prime}$ and $\lambda=\sqrt{3 b_{0} / 16 \pi^{2} \alpha^{\prime}}$ with the Regge slope $\alpha^{\prime}=1.04 \mathrm{GeV}^{-2}$. The function $v$ is

$$
v(x)=\frac{4 b_{0}}{\pi} \int_{0}^{\infty} \frac{d Q}{Q}\left(\rho\left(\vec{Q}^{2}\right)-\frac{K}{\vec{Q}^{2}}\right) \sin \left(\frac{Q}{\lambda} x\right)
$$

where $b_{0}=11-\frac{2}{3} N_{f}$ with the quark flavor number $N_{f}=4, K=3 / 16 \pi^{2} \alpha^{\prime}$ and $\rho\left(\vec{Q}^{2}\right)$ with gluon momentum $\vec{Q}$ is the physical running coupling constant given by Buchmüller and Tye [33]. The Schrödinger equation with the central spin-independent potential produces a radial wave function for the quark-antiquark relative motion of the $\pi$ and $\rho$ mesons while both the up-quark and down-quark masses take the value $0.33 \mathrm{GeV}$. The wave function leads to $0.6308 \mathrm{GeV}$ for the mass splitting between $\pi$ and $\rho$, versus the experimental value $0.6304 \mathrm{GeV}$, that is calculated with the spin-spin interaction

$$
V_{\mathrm{ss}}=-\frac{\vec{\lambda}_{a}}{2} \cdot \frac{\vec{\lambda}_{b}}{2} \frac{16 \pi^{2}}{25} \delta^{3}(\vec{r}) \frac{\vec{s}_{a} \cdot \vec{s}_{b}}{m_{a} m_{b}}+\frac{\vec{\lambda}_{a}}{2} \cdot \frac{\vec{\lambda}_{b}}{2} \frac{4 \pi}{25} \frac{1}{r} \frac{d^{2} v(\lambda r)}{d r^{2}} \frac{\vec{s}_{a} \cdot \vec{s}_{b}}{m_{a} m_{b}}
$$

where $\vec{s}_{a}\left(\vec{s}_{b}\right)$ and $m_{a}\left(m_{b}\right)$ are the spin and mass of the quark or antiquark constituent $a(b)$, respectively. If the strange quark mass is $0.53 \mathrm{GeV}$ and the radial quark-antiquark relative-motion wave functions of $K, K^{*}, \eta, \omega$ and $\phi$ are the same as that of $\pi$ and $\rho$, we get the mass splittings $m_{K^{*}}-m_{K}=0.3928 \mathrm{GeV}$ and $\frac{1}{3} m_{\omega}+\frac{2}{3} m_{\phi}-m_{\eta}=0.3733 \mathrm{GeV}$ in comparison to the experimental values $0.3963 \mathrm{GeV}$ and $0.3930 \mathrm{GeV}$, respectively. Here $m_{K}, m_{K^{*}}, m_{\eta}, m_{\omega}$ and $m_{\phi}$ are the masses of $K, K^{*}, \eta, \omega$ and $\phi$, respectively. The reasonable agreement between the theoretical results and the experimental data allows us 
to assume that all the mesons in the ground-state pseudoscalar octet and the ground-state vector nonet have the same spatial wave function of the quark-antiquark relative motion.

\section{Formulas}

In view of the fact that a meson consists of quark and antiquark constituents, $A\left(q_{1} \bar{q}_{1}\right)+$ $B\left(q_{2} \bar{q}_{2}\right) \rightarrow C\left(q_{1} \bar{q}_{2}\right)+D\left(q_{2} \bar{q}_{1}\right)$ is a 4 -to-4 constituent scattering that cannot be easily treated in QCD. However, in nonrelativistic dynamics what separates the quark-antiquark motion into their center-of-mass motion and relative motion, the scattering is reduced to a 2-to-2 scattering of two quark-antiquark pairs and the scattering problem is solved in a clear way from the S-matrix. S-matrix element for the reaction $A\left(q_{1} \bar{q}_{1}\right)+B\left(q_{2} \bar{q}_{2}\right) \rightarrow$ $C\left(q_{1} \bar{q}_{2}\right)+D\left(q_{2} \bar{q}_{1}\right)$ is

$$
S_{\mathrm{fi}}=\delta_{\mathrm{fi}}-2 \pi i \delta\left(E_{\mathrm{f}}-E_{\mathrm{i}}\right)<q_{1} \bar{q}_{2}, q_{2} \bar{q}_{1}\left|H_{\mathrm{I}}\right| q_{1} \bar{q}_{1}, q_{2} \bar{q}_{2}>
$$

where $H_{\mathrm{I}}$ is the interaction of two constituents each in an initial meson or in a final meson and $E_{\mathrm{i}}\left(E_{\mathrm{f}}\right)$ is the total energy of the two initial (final) mesons. The wave function of the initial mesons is

$$
\psi_{q_{1} \bar{q}_{1}, q_{2} \bar{q}_{2}}=\frac{e^{i \vec{P}_{q_{1} \bar{q}_{1}} \cdot \vec{R}_{q_{1} \bar{q}_{1}}}}{\sqrt{V}} \psi_{q_{1} \bar{q}_{1}}\left(\vec{r}_{q_{1} \bar{q}_{1}}\right) \frac{e^{i \vec{P}_{q_{2}} \bar{q}_{2} \cdot \vec{R}_{q_{2}} \bar{q}_{2}}}{\sqrt{V}} \psi_{q_{2} \bar{q}_{2}}\left(\vec{r}_{q_{2} \bar{q}_{2}}\right)
$$

which is normalized to one in volume $V$,

$$
\int d^{3} r_{q_{1} \bar{q}_{1}} \psi_{q_{1} \bar{q}_{1}}^{+}\left(\vec{r}_{q_{1} \bar{q}_{1}}\right) \psi_{q_{1} \bar{q}_{1}}\left(\vec{r}_{q_{1} \bar{q}_{1}}\right)=1
$$

and

$$
\int d^{3} r_{q_{2} \bar{q}_{2}} \psi_{q_{2} \bar{q}_{2}}^{+}\left(\vec{r}_{q_{2} \bar{q}_{2}}\right) \psi_{q_{2} \bar{q}_{2}}\left(\vec{r}_{q_{2} \bar{q}_{2}}\right)=1
$$

$\vec{P}_{q_{1} \bar{q}_{1}}\left(\vec{P}_{q_{2} \bar{q}_{2}}\right), \vec{R}_{q_{1} \bar{q}_{1}}\left(\vec{R}_{q_{2} \bar{q}_{2}}\right)$ and $\vec{r}_{q_{1} \bar{q}_{1}}\left(\vec{r}_{q_{2} \bar{q}_{2}}\right)$ are the total momentum, the center-of-mass coordinate and the relative coordinate of $q_{1}\left(q_{2}\right)$ and $\bar{q}_{1}\left(\bar{q}_{2}\right)$, respectively. The wave function of the final mesons is

$$
\psi_{q_{1} \bar{q}_{2}, q_{2} \bar{q}_{1}}=\frac{e^{i \vec{P}_{q_{1} \bar{q}_{2}} \cdot \vec{R}_{q_{1} \bar{q}_{2}}}}{\sqrt{V}} \psi_{q_{1} \bar{q}_{2}}\left(\vec{r}_{q_{1} \bar{q}_{2}}\right) \frac{e^{i \vec{P}_{q_{2}} \bar{q}_{1} \cdot \vec{R}_{q_{2} \bar{q}_{1}}}}{\sqrt{V}} \psi_{q_{2} \bar{q}_{1}}\left(\vec{r}_{q_{2} \bar{q}_{1}}\right)
$$

which is normalized to one in volume $V$,

$$
\int d^{3} r_{q_{1} \bar{q}_{2}} \psi_{q_{1} \bar{q}_{2}}^{+}\left(\vec{r}_{q_{1} \bar{q}_{2}}\right) \psi_{q_{1} \bar{q}_{2}}\left(\vec{r}_{q_{1} \bar{q}_{2}}\right)=1
$$

and

$$
\int d^{3} r_{q_{2} \bar{q}_{1}} \psi_{q_{2} \bar{q}_{1}}^{+}\left(\vec{r}_{q_{2} \bar{q}_{1}}\right) \psi_{q_{2} \bar{q}_{1}}\left(\vec{r}_{q_{2} \bar{q}_{1}}\right)=1
$$


$\vec{P}_{q_{1} \bar{q}_{2}}\left(\vec{P}_{q_{2} \bar{q}_{1}}\right), \vec{R}_{q_{1} \bar{q}_{2}}\left(\vec{R}_{q_{2} \bar{q}_{1}}\right)$ and $\vec{r}_{q_{1} \bar{q}_{2}}\left(\vec{r}_{q_{2} \bar{q}_{1}}\right)$ are the total momentum, the center-of-mass coordinate and the relative coordinate of $q_{1}\left(q_{2}\right)$ and $\bar{q}_{2}\left(\bar{q}_{1}\right)$, respectively. With the wave functions we obtain

$$
\begin{aligned}
<q_{1} \bar{q}_{2}, q_{2} \bar{q}_{1}\left|H_{\mathrm{I}}\right| q_{1} \bar{q}_{1}, q_{2} \bar{q}_{2}> & =\int d^{3} r_{q_{1} \bar{q}_{1}} d^{3} R_{q_{1} \bar{q}_{1}} d^{3} r_{q_{2} \bar{q}_{2}} d^{3} R_{q_{2} \bar{q}_{2}} \psi_{q_{1} \bar{q}_{2}, q_{2} \bar{q}_{1}}^{+} H_{I} \psi_{q_{1} \bar{q}_{1}, q_{2} \bar{q}_{2}} \\
& =(2 \pi)^{3} \delta\left(\vec{P}_{\mathrm{f}}-\vec{P}_{\mathrm{i}}\right) \frac{\mathcal{M}_{f_{i}}}{V^{2} \sqrt{2 E_{q_{1} \bar{q}_{1}} 2 E_{q_{2} \bar{q}_{2}} 2 E_{q_{1} \bar{q}_{2}} 2 E_{q_{2} \bar{q}_{1}}}}
\end{aligned}
$$

where $\vec{P}_{\mathrm{i}}\left(\vec{P}_{\mathrm{f}}\right)$ is the total three-dimensional momentum of the two initial (final) mesons, and $E_{q_{1} \bar{q}_{1}}, E_{q_{2} \bar{q}_{2}}, E_{q_{1} \bar{q}_{2}}$ and $E_{q_{2} \bar{q}_{1}}$ are the energies of the four mesons $q_{1} \bar{q}_{1}, q_{2} \bar{q}_{2}, q_{1} \bar{q}_{2}$ and $q_{2} \bar{q}_{1}$, respectively. The transition amplitude $\mathcal{M}_{f i}$ is

$$
\begin{aligned}
\mathcal{M}_{f i}= & \sqrt{2 E_{q_{1} \bar{q}_{1}} 2 E_{q_{2} \bar{q}_{2}} 2 E_{q_{1} \bar{q}_{2}} 2 E_{q_{2} \bar{q}_{1}}} \int d^{3} r_{q_{1} \bar{q}_{1}} d^{3} r_{q_{2} \bar{q}_{2}} d^{3} r_{q_{1} \bar{q}_{1}, q_{2} \bar{q}_{2}} \\
& \psi_{q_{1} \bar{q}_{2}}^{+}\left(\vec{r}_{q_{1} \bar{q}_{2}}\right) \psi_{q_{2} \bar{q}_{1}}^{+}\left(\vec{r}_{q_{2} \bar{q}_{1}}\right) H_{I} \psi_{q_{1} \bar{q}_{1}}\left(\vec{r}_{q_{1} \bar{q}_{1}}\right) \psi_{q_{2} \bar{q}_{2}}\left(\vec{r}_{q_{2} \bar{q}_{2}}\right) \mathrm{e}^{i \vec{p}_{q_{1}} \bar{q}_{1}, q_{2} \bar{q}_{2} \cdot \vec{r}_{q_{1}} \bar{q}_{1}, q_{2} \bar{q}_{2}-i \vec{p}_{q_{1}} \bar{q}_{2}, q_{2} \bar{q}_{1} \cdot \vec{r}_{q_{1} \bar{q}_{2}, q_{2} \bar{q}_{1}}}
\end{aligned}
$$

where $\vec{p}_{q_{1} \bar{q}_{1}, q_{2} \bar{q}_{2}}\left(\vec{p}_{q_{1} \bar{q}_{2}, q_{2} \bar{q}_{1}}\right)$ and $\vec{r}_{q_{1} \bar{q}_{1}, q_{2} \bar{q}_{2}}\left(\vec{r}_{q_{1} \bar{q}_{2}, q_{2} \bar{q}_{1}}\right)$ are the relative momentum and the relative coordinate of $q_{1} \bar{q}_{1}\left(q_{1} \bar{q}_{2}\right)$ and $q_{2} \bar{q}_{2}\left(q_{2} \bar{q}_{1}\right)$, respectively. $\psi_{a b}\left(\vec{r}_{a b}\right)$ is the wave function of the relative motion of constituents $a$ and $b$ in coordinate space. Further derivation similar to Ref. [35] leads to cross section for $A\left(q_{1} \bar{q}_{1}\right)+B\left(q_{2} \bar{q}_{2}\right) \rightarrow C\left(q_{1} \bar{q}_{2}\right)+D\left(q_{2} \bar{q}_{1}\right)$

$$
\begin{aligned}
\sigma= & \frac{(2 \pi)^{4}}{4 \sqrt{\left(P_{q_{1} \bar{q}_{1}} \cdot P_{q_{2} \bar{q}_{2}}\right)^{2}-m_{q_{1} \bar{q}_{1}}^{2} m_{q_{2} \bar{q}_{2}}^{2}}} \\
& \int \frac{d^{3} P_{q_{1} \bar{q}_{2}}}{(2 \pi)^{3} 2 E_{q_{1} \bar{q}_{2}}} \frac{d^{3} P_{q_{2} \bar{q}_{1}}}{(2 \pi)^{3} 2 E_{q_{2} \bar{q}_{1}}}\left|\mathcal{M}_{f i}\right|^{2} \delta\left(E_{\mathrm{f}}-E_{\mathrm{i}}\right) \delta\left(\vec{P}_{\mathrm{f}}-\vec{P}_{\mathrm{i}}\right)
\end{aligned}
$$

where $m_{q_{1} \bar{q}_{1}}\left(m_{q_{2} \bar{q}_{2}}\right)$ and $P_{q_{1} \bar{q}_{1}}=\left(E_{q_{1} \bar{q}_{1}}, \vec{P}_{q_{1} \bar{q}_{1}}\right)\left(P_{q_{2} \bar{q}_{2}}=\left(E_{q_{2} \bar{q}_{2}}, \vec{P}_{q_{2} \bar{q}_{2}}\right)\right)$ are the mass and the four-momentum of meson $A\left(q_{1} \bar{q}_{1}\right)\left(B\left(q_{2} \bar{q}_{2}\right)\right)$, respectively. Expressed in terms of the Mandelstam variables $s=\left(E_{q_{1} \bar{q}_{1}}+E_{q_{2} \bar{q}_{2}}\right)^{2}-\left(\vec{P}_{q_{1} \bar{q}_{1}}+\vec{P}_{q_{2} \bar{q}_{2}}\right)^{2}$ and $t=\left(E_{q_{1} \bar{q}_{1}}-E_{q_{1} \bar{q}_{2}}\right)^{2}-$ $\left(\vec{P}_{q_{1} \bar{q}_{1}}-\vec{P}_{q_{1} \bar{q}_{2}}\right)^{2}$, the cross section becomes

$$
\sigma=\frac{1}{32 \pi s} \frac{\left|\vec{P}^{\prime}(\sqrt{s})\right|}{|\vec{P}(\sqrt{s})|} \int_{0}^{\pi} d \theta\left|\mathcal{M}_{f i}(s, t)\right|^{2} \sin \theta
$$

where $\theta$ is the angle between $\vec{P}$ and $\vec{P}^{\prime}, \vec{P}=\vec{P}_{q_{1} \bar{q}_{1}}=-\vec{P}_{q_{2} \bar{q}_{2}}$ and $\vec{P}^{\prime}=\vec{P}_{q_{1} \bar{q}_{2}}=-\vec{P}_{q_{2} \bar{q}_{1}}$ in the center-of-momentum frame,

$$
\begin{aligned}
& |\vec{P}(\sqrt{s})|^{2}=\frac{1}{4 s}\left\{\left[s-\left(m_{q_{1} \bar{q}_{1}}^{2}+m_{q_{2} \bar{q}_{2}}^{2}\right)\right]^{2}-4 m_{q_{1} \bar{q}_{1}}^{2} m_{q_{2} \bar{q}_{2}}^{2}\right\}, \\
& \left|\vec{P}^{\prime}(\sqrt{s})\right|^{2}=\frac{1}{4 s}\left\{\left[s-\left(m_{q_{1} \bar{q}_{2}}^{2}+m_{q_{2} \bar{q}_{1}}^{2}\right)\right]^{2}-4 m_{q_{1} \bar{q}_{2}}^{2} m_{q_{2} \bar{q}_{1}}^{2}\right\},
\end{aligned}
$$


where $m_{q_{1} \bar{q}_{2}}$ and $m_{q_{2} \bar{q}_{1}}$ are the masses of the two mesons $C\left(q_{1} \bar{q}_{2}\right)$ and $D\left(q_{2} \bar{q}_{1}\right)$, respectively. Inelastic reactions are divided into two types: endothermic reactions which have $\vec{P} \neq 0$, $\vec{P}^{\prime}=0$ and $\sigma=0$ at threshold energy and exothermic reactions which possess $\vec{P}=0$, $\vec{P}^{\prime} \neq 0$ and $\sigma=+\infty$ at threshold energy.

Since quark or antiquark interchange and gluon exchange are two basic processes, "prior" diagrams in Fig. 1 and "post" diagrams in Fig. 2 are involved in the Bornorder meson-meson scattering $A+B \rightarrow C+D$. A scattering in the prior form means that the gluon exchange takes place prior to quark or antiquark interchange while the interchange in the post form is followed by the gluon exchange. The two forms may give different cross sections for a meson-meson scattering, which is the so-called post-prior discrepancy [36-38]. If the constituent-constituent interaction and the wave function $\psi_{a b}\left(\vec{r}_{a b}\right)$ determined by the interaction are used to calculate the transition amplitude, the post-prior discrepancy disappears, i.e. the results individually produced by the post form and the prior form are identical. The post-prior discrepancy may arise if the interaction used in the transition amplitude differs from the one used in the determination of $\psi_{a b}\left(\vec{r}_{a b}\right)$ or $\psi_{a b}\left(\vec{r}_{a b}\right)$ used in the transition amplitude is not that mesonic quark-antiquark relativemotion wave function determined by the Schrödinger equation. Cross section for a mesonmeson scattering is assumed to be the average of the two results obtained in the prior form and in the post form, respectively. $H_{\mathrm{I}}$ in the prior form is

$$
\begin{aligned}
H_{\mathrm{I}}^{\text {prior }}= & \int \frac{d^{3} Q}{(2 \pi)^{3}} V_{q_{1} \bar{q}_{2}}(\vec{Q}) \mathrm{e}^{i \vec{Q} \cdot \vec{r}_{q_{1}} \bar{q}_{2}}+\int \frac{d^{3} Q}{(2 \pi)^{3}} V_{\bar{q}_{1} q_{2}}(\vec{Q}) \mathrm{e}^{i \vec{Q} \cdot \vec{r}_{\bar{q}_{1} q_{2}}} \\
& +\int \frac{d^{3} Q}{(2 \pi)^{3}} V_{q_{1} q_{2}}(\vec{Q}) \mathrm{e}^{i \vec{Q} \cdot \vec{r}_{q_{1} q_{2}}}+\int \frac{d^{3} Q}{(2 \pi)^{3}} V_{\bar{q}_{1} \bar{q}_{2}}(\vec{Q}) \mathrm{e}^{i \vec{Q} \cdot \vec{r}_{\bar{q}_{1} \bar{q}_{2}}}
\end{aligned}
$$

where $\vec{Q}$ is the gluon momentum and $V_{a b}(\vec{Q})$ is the potential $V_{\mathrm{si}}+V_{\mathrm{ss}}$ in momentum space,

$$
\begin{aligned}
V_{a b}(\vec{Q})= & \frac{\vec{\lambda}_{a}}{2} \cdot \frac{\vec{\lambda}_{b}}{2} \frac{16 \pi^{2}}{\vec{Q}^{2}} \rho\left(\vec{Q}^{2}\right)-\frac{\vec{\lambda}_{a}}{2} \cdot \frac{\vec{\lambda}_{b}}{2} \frac{16 \pi^{2}}{25} \frac{\vec{s}_{a} \cdot \vec{s}_{b}}{m_{a} m_{b}} \\
& +\frac{\vec{\lambda}_{a}}{2} \cdot \frac{\vec{\lambda}_{b}}{2} \frac{16 \pi^{2} \lambda}{25 Q} \int_{0}^{+\infty} d x \frac{d^{2} v(x)}{d x^{2}} \sin \left(\frac{Q}{\lambda} x\right) \frac{\vec{s}_{a} \cdot \vec{s}_{b}}{m_{a} m_{b}}
\end{aligned}
$$

The transition amplitude in the prior form is

$$
\begin{aligned}
\mathcal{M}_{f i}^{\text {prior }}= & \sqrt{2 E_{q_{1} \bar{q}_{1}} 2 E_{q_{2} \bar{q}_{2}} 2 E_{q_{1} \bar{q}_{2}} 2 E_{q_{2} \bar{q}_{1}}} \int \frac{d^{3} p_{q_{1} \bar{q}_{2}}}{(2 \pi)^{3}} \frac{d^{3} p_{q_{2} \bar{q}_{1}}}{(2 \pi)^{3}} \\
& \psi_{q_{1} \bar{q}_{2}}^{+}\left(\vec{p}_{q_{1} \bar{q}_{2}}\right) \psi_{q_{2} \bar{q}_{1}}^{+}\left(\vec{p}_{q_{2} \bar{q}_{1}}\right)\left(V_{q_{1} \bar{q}_{2}}+V_{\bar{q}_{1} q_{2}}+V_{q_{1} q_{2}}+V_{\bar{q}_{1} \bar{q}_{2}}\right) \psi_{q_{1} \bar{q}_{1}}\left(\vec{p}_{q_{1} \bar{q}_{1}}\right) \psi_{q_{2} \bar{q}_{2}}\left(\vec{p}_{q_{2} \bar{q}_{2}}\right)
\end{aligned}
$$

where $\psi_{a b}\left(\vec{p}_{a b}\right)$ is the wave function of the relative motion of constituents $a$ and $b$ in momentum space and satisfies $\int \frac{d^{3} p_{a b}}{(2 \pi)^{3}} \psi_{a b}^{+}\left(\vec{p}_{a b}\right) \psi_{a b}\left(\vec{p}_{a b}\right)=1 . \vec{p}_{a b}$ is the relative momentum 
of $a$ and $b . H_{\mathrm{I}}$ in the post form is

$$
\begin{aligned}
H_{\mathrm{I}}^{\text {post }}= & \int \frac{d^{3} Q}{(2 \pi)^{3}} V_{q_{1} \bar{q}_{1}}(\vec{Q}) \mathrm{e}^{i \vec{Q} \cdot \vec{r}_{q_{1}} \bar{q}_{1}}+\int \frac{d^{3} Q}{(2 \pi)^{3}} V_{\bar{q}_{2} q_{2}}(\vec{Q}) \mathrm{e}^{i \vec{Q} \cdot \vec{r}_{\bar{q}_{2} q_{2}}} \\
& +\int \frac{d^{3} Q}{(2 \pi)^{3}} V_{q_{1} q_{2}}(\vec{Q}) \mathrm{e}^{i \vec{Q} \cdot \vec{r}_{q_{1} q_{2}}}+\int \frac{d^{3} Q}{(2 \pi)^{3}} V_{\bar{q}_{1} \bar{q}_{2}}(\vec{Q}) \mathrm{e}^{i \vec{Q} \cdot \vec{r}_{\bar{q}_{1} \bar{q}_{2}}}
\end{aligned}
$$

which leads to the transition amplitude in the post form

$$
\begin{aligned}
\mathcal{M}_{f i}^{\text {post }}= & \sqrt{2 E_{q_{1} \bar{q}_{1}} 2 E_{q_{2} \bar{q}_{2}} 2 E_{q_{1} \bar{q}_{2}} 2 E_{q_{2} \bar{q}_{1}}} \\
& \left(\int \frac{d^{3} p_{q_{1} \bar{q}_{1}}}{(2 \pi)^{3}} \frac{d^{3} p_{q_{1} \bar{q}_{2}}}{(2 \pi)^{3}} \psi_{q_{1} \bar{q}_{2}}^{+}\left(\vec{p}_{q_{1} \bar{q}_{2}}\right) \psi_{q_{2} \bar{q}_{1}}^{+}\left(\vec{p}_{q_{2} \bar{q}_{1}}\right) V_{q_{1} \bar{q}_{1}} \psi_{q_{1} \bar{q}_{1}}\left(\vec{p}_{q_{1} \bar{q}_{1}}\right) \psi_{q_{2} \bar{q}_{2}}\left(\vec{p}_{q_{2} \bar{q}_{2}}\right)\right. \\
& +\int \frac{d^{3} p_{q_{2}} \bar{q}_{2}}{(2 \pi)^{3}} \frac{d^{3} p_{q_{2} \bar{q}_{1}}}{(2 \pi)^{3}} \psi_{q_{1} \bar{q}_{2}}^{+}\left(\vec{p}_{q_{1} \bar{q}_{2}}\right) \psi_{q_{2} \bar{q}_{1}}^{+}\left(\vec{p}_{q_{2} \bar{q}_{1}}\right) V_{\bar{q}_{2} q_{2}} \psi_{q_{1} \bar{q}_{1}}\left(\vec{p}_{q_{1} \bar{q}_{1}}\right) \psi_{q_{2} \bar{q}_{2}}\left(\vec{p}_{q_{2} \bar{q}_{2}}\right) \\
& \left.+\int \frac{d^{3} p_{q_{1} \bar{q}_{2}}}{(2 \pi)^{3}} \frac{d^{3} p_{q_{2} \bar{q}_{1}}}{(2 \pi)^{3}} \psi_{q_{1} \bar{q}_{2}}^{+}\left(\vec{p}_{q_{1} \bar{q}_{2}}\right) \psi_{q_{2} \bar{q}_{1}}^{+}\left(\vec{p}_{q_{2} \bar{q}_{1}}\right)\left(V_{q_{1} q_{2}}+V_{\bar{q}_{1} \bar{q}_{2}}\right) \psi_{q_{1} \bar{q}_{1}}\left(\vec{p}_{q_{1} \bar{q}_{1}}\right) \psi_{q_{2} \bar{q}_{2}}\left(\vec{p}_{q_{2} \bar{q}_{2}}\right)\right)
\end{aligned}
$$

Let $\sigma\left(S, m_{S}, \sqrt{s}\right) \equiv \sigma$ denote the cross section corresponding to a component $m_{S}$ of the total spin $S$ of the two incoming mesons in the following eleven channels of $A\left(q_{1} \bar{q}_{1}\right)+$ $B\left(q_{2} \bar{q}_{2}\right) \rightarrow C\left(q_{1} \bar{q}_{2}\right)+D\left(q_{2} \bar{q}_{1}\right)$

$$
\begin{aligned}
& (1) q_{1} \bar{q}_{1}\left(S_{A}=0\right)+q_{2} \bar{q}_{2}\left(S_{B}=0\right) \rightarrow q_{1} \bar{q}_{2}\left(S_{C}=0\right)+q_{2} \bar{q}_{1}\left(S_{D}=0\right) ; S=0 ; \\
& (2) q_{1} \bar{q}_{1}\left(S_{A}=0\right)+q_{2} \bar{q}_{2}\left(S_{B}=0\right) \rightarrow q_{1} \bar{q}_{2}\left(S_{C}=1\right)+q_{2} \bar{q}_{1}\left(S_{D}=1\right) ; S=0 ; \\
& \text { (3) } q_{1} \bar{q}_{1}\left(S_{A}=0\right)+q_{2} \bar{q}_{2}\left(S_{B}=1\right) \rightarrow q_{1} \bar{q}_{2}\left(S_{C}=0\right)+q_{2} \bar{q}_{1}\left(S_{D}=1\right) ; S=1 ; \\
& \text { (4) } q_{1} \bar{q}_{1}\left(S_{A}=0\right)+q_{2} \bar{q}_{2}\left(S_{B}=1\right) \rightarrow q_{1} \bar{q}_{2}\left(S_{C}=1\right)+q_{2} \bar{q}_{1}\left(S_{D}=0\right) ; S=1 ; \\
& \text { (5) } q_{1} \bar{q}_{1}\left(S_{A}=0\right)+q_{2} \bar{q}_{2}\left(S_{B}=1\right) \rightarrow q_{1} \bar{q}_{2}\left(S_{C}=1\right)+q_{2} \bar{q}_{1}\left(S_{D}=1\right) ; S=1 ; \\
& \text { (6) } q_{1} \bar{q}_{1}\left(S_{A}=1\right)+q_{2} \bar{q}_{2}\left(S_{B}=1\right) \rightarrow q_{1} \bar{q}_{2}\left(S_{C}=0\right)+q_{2} \bar{q}_{1}\left(S_{D}=0\right) ; S=0 ; \\
& \text { (7) } q_{1} \bar{q}_{1}\left(S_{A}=1\right)+q_{2} \bar{q}_{2}\left(S_{B}=1\right) \rightarrow q_{1} \bar{q}_{2}\left(S_{C}=0\right)+q_{2} \bar{q}_{1}\left(S_{D}=1\right) ; S=1 ; \\
& \text { (8) } q_{1} \bar{q}_{1}\left(S_{A}=1\right)+q_{2} \bar{q}_{2}\left(S_{B}=1\right) \rightarrow q_{1} \bar{q}_{2}\left(S_{C}=1\right)+q_{2} \bar{q}_{1}\left(S_{D}=0\right) ; S=1 ; \\
& \text { (9) } q_{1} \bar{q}_{1}\left(S_{A}=1\right)+q_{2} \bar{q}_{2}\left(S_{B}=1\right) \rightarrow q_{1} \bar{q}_{2}\left(S_{C}=1\right)+q_{2} \bar{q}_{1}\left(S_{D}=1\right) ; S=0 ; \\
& \text { (10) } q_{1} \bar{q}_{1}\left(S_{A}=1\right)+q_{2} \bar{q}_{2}\left(S_{B}=1\right) \rightarrow q_{1} \bar{q}_{2}\left(S_{C}=1\right)+q_{2} \bar{q}_{1}\left(S_{D}=1\right) ; S=1 ; \\
& \text { (11) } q_{1} \bar{q}_{1}\left(S_{A}=1\right)+q_{2} \bar{q}_{2}\left(S_{B}=1\right) \rightarrow q_{1} \bar{q}_{2}\left(S_{C}=1\right)+q_{2} \bar{q}_{1}\left(S_{D}=1\right) ; S=2 ;
\end{aligned}
$$

where the quantities $S_{A}, S_{B}, S_{C}$ and $S_{D}$ in the parentheses are meson spins. In fact, $\sigma\left(S, m_{S}, \sqrt{s}\right)$ is independent of $m_{S}$ and is calculated at any value subject to the condition 
$-S \leq m_{S} \leq S$. When the orbital angular momenta of the four mesons $A, B, C$ and $D$ are all zero, the unpolarized cross section is

$$
\sigma^{\mathrm{unpol}}(\sqrt{s})=\frac{1}{\left(2 S_{A}+1\right)\left(2 S_{B}+1\right)} \sum_{S}(2 S+1) \sigma\left(S, m_{S}, \sqrt{s}\right)
$$

where $S$ takes values that are allowed by $\left|S_{A}-S_{B}\right| \leq S \leq S_{A}+S_{B}$ and $\left|S_{C}-S_{D}\right| \leq$ $S \leq S_{C}+S_{D}$

\section{Numerical results and discussions}

The transition amplitude $\mathcal{M}_{f i}$ is obtained from meson wave functions of which each is the product of quark-antiquark relative-motion, spin, flavor and color wave functions. The quark-antiquark relative-motion wave function $\psi_{a b}\left(\vec{p}_{a b}\right)$ is the Fourier transform of the wave function $\psi_{a b}\left(\vec{r}_{a b}\right)$ what is obtained by the Schrödinger equation with the BuchmüllerTye potential. $\psi_{q_{1} \bar{q}_{1}}\left(\vec{p}_{q_{1} \bar{q}_{1}}\right), \psi_{q_{2} \bar{q}_{2}}\left(\vec{p}_{q_{2} \bar{q}_{2}}\right), \psi_{q_{1} \bar{q}_{2}}\left(\vec{p}_{q_{1} \bar{q}_{2}}\right)$ and $\psi_{q_{2} \bar{q}_{1}}\left(\vec{p}_{q_{2} \bar{q}_{1}}\right)$ take the same form. The matrix elements of $\vec{s}_{a} \cdot \vec{s}_{b}$ are derived for the eleven channels. The transition amplitude is calculated in the center-of-momentum frame of the two initial mesons where

$$
4 \sqrt{E_{q_{1} \bar{q}_{1}} E_{q_{2} \bar{q}_{2}} E_{q_{1} \bar{q}_{2}} E_{q_{2} \bar{q}_{1}}}=\frac{1}{s} \sqrt{\left[s^{2}-\left(m_{q_{1} \bar{q}_{1}}^{2}-m_{q_{2} \bar{q}_{2}}^{2}\right)^{2}\right]\left[s^{2}-\left(m_{q_{1} \bar{q}_{2}}^{2}-m_{q_{2} \bar{q}_{1}}^{2}\right)^{2}\right]} .
$$

4.1. Elastic phase shifts for $I=2 \pi \pi$ and $I=3 / 2 K \pi$ scattering

The phase shift formula is [17]

$$
\delta_{l}=-\frac{2 \pi^{2}|\vec{P}| E_{q_{1} \bar{q}_{1}} E_{q_{2} \bar{q}_{2}}}{E_{q_{1} \bar{q}_{1}}+E_{q_{2} \bar{q}_{2}}} \int_{-1}^{1} T_{f i} P_{l}\left(x^{\prime}\right) d x^{\prime},
$$

where $P_{l}\left(x^{\prime}\right)$ with $x^{\prime}=\cos \theta$ is the Legendre polynomial. $T_{f i}$ is related to the transition amplitudes in the prior form and in the post form

$$
T_{f i}=\frac{1}{(2 \pi)^{3} \sqrt{2 E_{q_{1} \bar{q}_{1}} 2 E_{q_{2} \bar{q}_{2}} 2 E_{q_{1} \bar{q}_{2}} 2 E_{q_{2} \bar{q}_{1}}}} \frac{\mathcal{M}_{f i}^{\text {prior }}+\mathcal{M}_{f i}^{\text {post }}}{2}
$$

The S-wave elastic phase shifts for $I=2 \pi \pi$ and $I=3 / 2 K \pi$ scattering given by Eq. (24) are plotted as solid curves in Figs. 3 and 4, respectively. Our theoretical values for the $I=2 \pi \pi$ elastic scattering are in good agreement with the experimental data. Our theoretical values for the $I=3 / 2 K \pi$ cannot completely match the poor experimental data but agree with from threshold energy to $\sqrt{s}=1.3 \mathrm{GeV}$ what is the region accessible in hadronic matter.

The solid curve is obtained when physical meson masses and flavor-symmetry breaking are used and the quark-antiquark relative-motion wave functions of $K$ and $\pi$ are taken to 
be identical. The nonstrange and strange quark masses remain as the values determined by ground-state meson spectroscopy via the Schrödinger equation with the BuchmüllerTye potential in Section 2. Squares and circles in Fig. 4 are data of Ref. [14] and Ref. [15], respectively. The two measurements may give different data when the center-ofmass energy of $K$ and $\pi$ is larger than $1.2 \mathrm{GeV}$. The possible discrepancy requires further examination of $K \pi$ phase shift in both experiment and theory. This is one reason why we calculate the $I=3 / 2 K \pi$ phase shift.

The transition amplitude is calculated with the quark-antiquark relative-motion wave functions what are solutions of the Schrödinger equation with the central spin-independent potential. But the interaction employed in the transition amplitude contains both the central spin-independent potential and the spin-spin term. Then the wave functions are not exact with respect to the interaction. Therefore, the post-prior discrepancy should come from the approximate wave functions and the spin-spin term. Fortunately, in the elastic scatterings where meson masses do not change, the spin-spin term gives almost the same magnitudes to the transition amplitudes individually obtained in the post form and in the prior form, and the post-prior discrepancy is completely negligible. For inelastic scatterings where meson masses change, the spin-spin term produces different magnitudes, hence the post-prior discrepancy exists.

4.2. Cross sections for inelastic scatterings of nonstrange mesons

Unpolarized cross sections for the $I=2 \pi \pi \rightarrow \rho \rho$ and $I=2 \rho \rho \rightarrow \pi \pi$ are shown in Fig. 5. The former has a maximum cross section of $0.47 \mathrm{mb}$ at $\sqrt{s}=1.79 \mathrm{GeV}$. Since no $s$-channel resonances contribute significantly to the reaction $\pi^{+} \pi^{-} \leftrightarrow \omega \omega$ as well as no quark-antiquark annihilation happens, we can use the quark-interchange mechanism to calculate the cross section for the reaction $\pi^{+} \pi^{-} \leftrightarrow \omega \omega$. Flavor matrix elements for the $I=2 \pi \pi \leftrightarrow \rho \rho$ and $\pi^{+} \pi^{-} \leftrightarrow \omega \omega$ inelastic scatterings are 1 and $-1 / 2$, respectively. The discrepancy of the cross sections for the two reactions comes from their different flavor matrix elements while their color and spin matrix elements are not different and the integration over relative-momentum variables in $\mathcal{M}_{f i}^{\text {prior }}$ and $\mathcal{M}_{f i}^{\text {post }}$ with nearly equal $\rho$ and $\omega$ masses offers almost the same values. Then the unpolarized cross section for the reaction $\pi^{+} \pi^{-} \leftrightarrow \omega \omega$ is one fourth of the one for the reaction of $I=2 \pi \pi \leftrightarrow \rho \rho$.

Unpolarized cross sections for the $I=2 \pi \rho \rightarrow \rho \rho$ and $I=2 \rho \rho \rightarrow \pi \rho$ are shown in Fig. 6. The endothermic reaction has a maximum cross section of $0.73 \mathrm{mb}$ at $\sqrt{s}=$ $1.86 \mathrm{GeV}$. The quark-interchange mechanism can also be applied to calculate cross sections for $\pi^{+} \rho^{-} \leftrightarrow \omega \omega$ and $\pi^{-} \rho^{+} \leftrightarrow \omega \omega$ inelastic scatterings. According to flavor matrix elements, the unpolarized cross section for the reaction $\pi^{+} \rho^{-} \leftrightarrow \omega \omega$ or $\pi^{-} \rho^{+} \leftrightarrow \omega \omega$ is one 
fourth of the one for the reaction of $I=2 \pi \rho \leftrightarrow \rho \rho$. The two reactions of $I=2 \pi \pi \rightarrow \rho \rho$ and $I=2 \pi \rho \rightarrow \rho \rho$ have the same threshold energy but the latter has larger cross section than the former. Such difference originates from the physical masses of initial mesons and the total spins $S=0$ for the $I=2 \pi \pi \rightarrow \rho \rho$ and $S=1$ for the $I=2 \pi \rho \rightarrow \rho \rho$.

4.3. Cross sections for inelastic scatterings of strange mesons

Unpolarized cross sections for the $I=1 K K \leftrightarrow K^{*} K^{*}$ and $I=1 K K^{*} \leftrightarrow K^{*} K^{*}$ inelastic scatterings are shown in Figs. 7 and 8, respectively. Since the two reactions have the same threshold energy, they can be compared. The reaction of $I=1 K K \rightarrow K^{*} K^{*}$ has a maximum cross section of $0.60 \mathrm{mb}$ at $\sqrt{s}=1.94 \mathrm{GeV}$ while the reaction of $I=$ $1 K K^{*} \rightarrow K^{*} K^{*}$ has a maximum cross section of $0.84 \mathrm{mb}$ at $\sqrt{s}=2.09 \mathrm{GeV}$. Given a center-of-mass energy $\sqrt{s}$, according to Eqs. (15) and (16) the outgoing $K^{*}$ mesons of the two reactions have the same momentum $\left|\vec{P}^{\prime}\right|$, but the $K$ mesons in the reaction of $I=1 K K \rightarrow K^{*} K^{*}$ have larger momentum $|\vec{P}|$ than the initial $K^{*}$ and $K$ mesons in the reaction of $I=1 K K^{*} \rightarrow K^{*} K^{*}$. Then the factor $\left|\vec{P}^{\prime}\right| /|\vec{P}|$ what has a smaller value for the former than the one for the latter gives a smaller cross section to the former than the latter in spite of the difference generated by the different total spins of the two reactions. Among inelastic scatterings of strange mesons, the reactions $K K \leftrightarrow K K^{*}$ are forbidden since the total spin of the two incoming mesons is not equal to that of the two outgoing mesons.

4.4. Cross sections for inelastic scatterings of nonstrange mesons by strange mesons

This subsection contributes to the $I=3 / 2$ inelastic scatterings between one of $\pi$ and $\rho$ and one of $K$ and $K^{*}$. Figs. 9, 10 and 11 individually show unpolarized cross sections for the inelastic scatterings of $I=3 / 2 \pi K \leftrightarrow \rho K^{*}, I=3 / 2 \pi K^{*} \leftrightarrow \rho K^{*}$ and $I=3 / 2 \rho K \leftrightarrow \rho K^{*}$ which have the same threshold energy. The reaction of $I=$ $3 / 2 \pi K \rightarrow \rho K^{*}\left(I=3 / 2 \pi K^{*} \rightarrow \rho K^{*}, I=3 / 2 \rho K \rightarrow \rho K^{*}\right)$ has a maximum cross section of $0.52 \mathrm{mb}(0.47 \mathrm{mb}, 0.51 \mathrm{mb})$ at $\sqrt{s}=1.76 \mathrm{GeV}(1.94 \mathrm{GeV}, 1.99 \mathrm{GeV})$. The maximum cross sections for the three reactions are comparable to those shown in the last two subsections. Attractively, the reaction of $I=3 / 2 \pi K^{*} \rightarrow \rho K$ exhibited in Fig. 12 has a maximum cross section of $1.41 \mathrm{mb}$ at $\sqrt{s}=1.42 \mathrm{GeV}$. The maximum cross section for this reaction is quite lager that any maximum cross section seen before. This case is caused by the small threshold energy $1.266 \mathrm{GeV}$ and the samll difference $0.234 \mathrm{GeV}$ of the mass sums of the final mesons and of the initial mesons. Therefore, the reaction of $I=3 / 2 \pi K^{*} \rightarrow \rho K$ is most important among the endothermic nonresonant reactions. 
4.5. Cross sections for $\phi$ meson in collisions with $\pi$ and $\rho$ mesons

Unpolarized cross sections for the $I=1 \pi \phi \rightarrow K K^{*}$ (or $K^{*} K$ ) and the $I=1 \pi \phi \rightarrow$ $K^{*} K^{*}$ are shown in Figs. 13 and 14, respectively. $K$ denotes either a kaon or an antikaon as appropriate. These reactions are endothermic. The reaction of $I=1 \pi \phi \rightarrow K K^{*}$ (or $K^{*} K$ ) has a maximum cross section of $0.99 \mathrm{mb}$ at $\sqrt{s}=1.51 \mathrm{GeV}$ near the threshold energy. The cross section for the reaction of $I=1 \pi \phi \rightarrow K^{*} K^{*}$ features a wide shape with a maximum of $0.65 \mathrm{mb}$ at $\sqrt{s}=2.11 \mathrm{GeV}$. The exothermic reaction $\pi \phi \rightarrow K K$ is forbidden since the total spin of the two incoming mesons does not equal that of the two outgoing mesons.

Unpolarized cross sections for the reactions of $I=1 \rho \phi \rightarrow K K, I=1 \rho \phi \rightarrow K K^{*}$ (or $\left.K^{*} K\right)$ and $I=1 \rho \phi \rightarrow K^{*} K^{*}$ are shown in the upper, middle and lower panels in Fig. 15, respectively. These exothermic reactions have the same threshold energy which equals the sum of the physical $\rho$ and $\phi$ masses. The cross sections for the $I=1 \rho \phi \rightarrow K K$ and the $I=1 \rho \phi \rightarrow K K^{*}$ (or $K^{*} K$ ) decreases very rapidly from the threshold energy but the cross section for the $I=1 \rho \phi \rightarrow K^{*} K^{*}$ decreases slowly from $\sqrt{s}=2 \mathrm{GeV}$.

Some calculations of transport equations utilize a constant cross section for the scattering of $\phi$ and a meson as an input. A choice on the value of the constant cross section lacks of direct experimental and theoretical support. To guide the choice, we evaluate the constant from the meson- $\phi$ cross section for a reaction that we have studied so far. We define the average cross section for $\phi$ in collision with a meson

$$
\left\langle\sigma_{m \phi}\right\rangle=\frac{g_{m} \int \frac{d^{3} k_{1}}{(2 \pi)^{3}} f_{m}\left(k_{1}\right) \frac{d^{3} k_{2}}{(2 \pi)^{3}} f_{\phi}\left(k_{2}\right) \sigma_{m \phi}(\sqrt{s})}{g_{m} \int \frac{d^{3} k_{1}}{(2 \pi)^{3}} f_{m}\left(k_{1}\right) \int \frac{d^{3} k_{2}}{(2 \pi)^{3}} f_{\phi}\left(k_{2}\right)},
$$

where $g_{m}$ is the spin degeneracy factor of the meson, $f_{m}$ and $f_{\phi}$ are the momentum distributions of the meson and $\phi$, respectively; $\sigma_{m \phi}(\sqrt{s})$ is the $\sqrt{s}$-dependent meson- $\phi$ cross section. $\left\langle\sigma_{m \phi}\right\rangle$ is independent of $\sqrt{s}$ that relates to $k_{1}$ and $k_{2}$ and is suggested as the constant.

Table 1: Average values for $\phi$ meson in collisions with $\pi$ and $\rho$ mesons.

\begin{tabular}{ccc}
\hline Channel & $\left\langle\sigma_{m \phi}\right\rangle(\mathrm{mb})$ & $\left\langle v_{\mathrm{rel}} \sigma_{m \phi}\right\rangle(\mathrm{mb})$ \\
\hline$I=1 \pi \phi \rightarrow K K^{*}\left(\right.$ or $\left.K^{*} K\right)$ & 0.365 & 0.374 \\
$I=1 \pi \phi \rightarrow K^{*} K^{*}$ & 0.056 & 0.073 \\
$I=1 \rho \phi \rightarrow K K$ & 0.590 & 0.248 \\
$I=1 \rho \phi \rightarrow K K^{*}\left(\right.$ or $\left.K^{*} K\right)$ & 0.624 & 0.284 \\
$I=1 \rho \phi \rightarrow K^{*} K^{*}$ & 2.162 & 1.235 \\
\hline
\end{tabular}


Since $\phi$ mesons in hadronic matter may be in thermal equilibrium [32,39], we assume $f_{m}\left(k_{1}\right)=e^{-E_{m} / T}$ with the meson energy $E_{m}$ and $f_{\phi}\left(k_{2}\right)=e^{-E_{\phi} / T}$ with the $\phi$ energy $E_{\phi}$. At temperature $T=0.15 \mathrm{GeV}$, average cross sections are listed in Table 11. The results for the reactions of $I=1 \pi \phi \rightarrow K^{*} K^{*}$ and $I=1 \rho \phi \rightarrow K^{*} K^{*}$ need to be specified. Since the difference of the total masses of the initial mesons and of the final mesons in the $I=1 \pi \phi \rightarrow K^{*} K^{*}$ reaction is $0.63 \mathrm{GeV}$, what is much larger than the temperature, the momenta of $\pi$ and $\phi$ that can trigger the reaction are at least $0.59 \mathrm{GeV}$ much larger than $T$. Then $f_{m} \ll 1$ and $f_{\phi} \ll 1$ leads to the very small average cross section 0.056 mb. Since the unpolarized cross section for the $I=1 \rho \phi \rightarrow K^{*} K^{*}$ decreases slowly in the region that is not far away from the threshold energy, $f_{m}$ and $f_{\phi}$ at low momenta of $\rho$ and $\phi$ produce the particularly large average cross section for the $I=1 \rho \phi \rightarrow K^{*} K^{*}$, which indicates that the reaction is important.

In the last column is listed another interesting thermal-averaged quantity

$$
\left\langle v_{\mathrm{rel}} \sigma_{m \phi}\right\rangle=\frac{g_{m} \int \frac{d^{3} k_{1}}{(2 \pi)^{3}} f_{m}\left(k_{1}\right) \frac{d^{3} k_{2}}{(2 \pi)^{3}} f_{\phi}\left(k_{2}\right) v_{\mathrm{rel}} \sigma_{m \phi}(\sqrt{s})}{g_{m} \int \frac{d^{3} k_{1}}{(2 \pi)^{3}} f_{m}\left(k_{1}\right) \int \frac{d^{3} k_{2}}{(2 \pi)^{3}} f_{\phi}\left(k_{2}\right)},
$$

where $v_{\text {rel }}$ is the relative velocity of the meson and $\phi$. The orders of $\left\langle v_{\text {rel }} \sigma_{m \phi}\right\rangle$ are identical with the orders of $\left\langle\sigma_{m \phi}\right\rangle$. The scales $\left\langle\sigma_{m \phi}\right\rangle=0.5 \mathrm{mb}$ and $\left\langle v_{\text {rel }} \sigma_{m \phi}\right\rangle=0.3 \mathrm{mb}$ can be useful in spite of the types of reactions.

\subsection{General discussions}

Maximum cross sections for ten endothermic reactions, corresponding center-of-mass energy $\sqrt{s}$, initial meson momentum $|\vec{P}|$, final meson momentum $\left|\vec{P}^{\prime}\right|$ and $\frac{1}{s} \frac{\left|\overrightarrow{P^{\prime}}\right|}{|\vec{P}|}$ are listed in Table 2. The reactions but the $I=\frac{3}{2} \pi K^{*} \rightarrow \rho K$ and the $I=1 \pi \phi \rightarrow K K^{*}$ have small differences of the initial meson momenta. If the final meson momentum is large (small), the factor $\frac{1}{s} \frac{\left|\vec{P}^{\prime}\right|}{|\vec{P}|}$ is large (small), but the squared transition amplitude $\left|\mathcal{M}_{f i}\right|^{2}$ is small (large) since $\left|\mathcal{M}_{f i}\right|^{2}$ decreases with increasing $\left|\vec{P}^{\prime}\right|$. The product of $\frac{1}{s} \frac{\left|\vec{P}^{\prime}\right|}{|\vec{P}|}$ and $\left|\mathcal{M}_{f i}\right|^{2}$ in the cross section formula (Eq. (14)) changes slowly with respect to the variation of final meson momentum from one to another reaction. We can thus understand why the eight endothermic reactions have similar maximum cross sections, i.e. from $0.45 \mathrm{mb}$ to 0.85 mb. Compared to the eight reactions, the $I=\frac{3}{2} \pi K^{*} \rightarrow \rho K$ and the $I=1 \pi \phi \rightarrow K K^{*}$ have larger $\frac{1}{s} \frac{\left|\vec{P}^{\prime}\right|}{|\vec{P}|}$ and smaller $|\vec{P}|$ and $\left|\vec{P}^{\prime}\right|$ which leads to larger $\left|\mathcal{M}_{f i}\right|^{2}$. This accounts for the larger cross sections of the two reactions than the eight reactions.

When $\sqrt{s}$ increases, $\int_{0}^{\pi} d \theta \sin \theta\left|\mathcal{M}_{f i}\right|^{2}$ decreases slowly near the threshold energy and rapidly in the other region, and by contrast the factor $\frac{1}{s} \frac{\left|\vec{P}^{\prime}\right|}{|\vec{P}|}$ increases very rapidly and decreases moderately. The peak of $\frac{1}{s} \frac{\left|\vec{P}^{\prime}\right|}{|\vec{P}|}$ locates in the slowly-changing region of 
Table 2: Maximum cross sections $\sigma_{\max }$ and corresponding variables.

\begin{tabular}{cccccc}
\hline reaction & $\sqrt{s}(\mathrm{GeV})$ & $\sigma_{\max }(\mathrm{mb})$ & $|\vec{P}|(\mathrm{GeV})$ & $\left|\vec{P}^{\prime}\right|(\mathrm{GeV})$ & $\frac{1}{s} \frac{\left|\vec{P}^{\prime}\right|}{|\vec{P}|}\left(\mathrm{GeV}^{-2}\right)$ \\
\hline$I=2 \pi \pi \rightarrow \rho \rho$ & 1.79 & 0.47 & 0.884 & 0.456 & 0.161 \\
$I=2 \pi \rho \rightarrow \rho \rho$ & 1.86 & 0.73 & 0.763 & 0.522 & 0.197 \\
$I=1 K K \rightarrow K^{*} K^{*}$ & 1.94 & 0.60 & 0.834 & 0.377 & 0.120 \\
$I=1 K K^{*} \rightarrow K^{*} K^{*}$ & 2.09 & 0.84 & 0.766 & 0.541 & 0.162 \\
$I=\frac{3}{2} \pi K \rightarrow \rho K^{*}$ & 1.76 & 0.52 & 0.804 & 0.286 & 0.115 \\
$I=\frac{3}{2} \pi K^{*} \rightarrow \rho K^{*}$ & 1.94 & 0.47 & 0.756 & 0.498 & 0.175 \\
$I=\frac{3}{2} \rho K \rightarrow \rho K^{*}$ & 1.99 & 0.51 & 0.760 & 0.545 & 0.181 \\
$I=\frac{3}{2} \pi K^{*} \rightarrow \rho K^{*}$ & 1.42 & 1.41 & 0.413 & 0.316 & 0.379 \\
$I=1 \pi \phi \rightarrow K K^{*}$ & 1.51 & 0.99 & 0.394 & 0.285 & 0.318 \\
$I=1 \pi \phi \rightarrow K^{*} K^{*}$ & 2.11 & 0.65 & 0.801 & 0.560 & 0.157 \\
\hline
\end{tabular}

$\int_{0}^{\pi} d \theta \sin \theta\left|\mathcal{M}_{f i}\right|^{2}$. In case $\int_{0}^{\pi} d \theta \sin \theta\left|\mathcal{M}_{f i}\right|^{2}$ for an endothermic reaction decreases very slowly near the threshold energy, the cross section for the reaction takes its maximum at the same $\sqrt{s}$ as $\frac{1}{s} \frac{\left|\vec{P}^{\prime}\right|}{|\vec{P}|}$ does. Otherwise the peak of cross section locates at the left of but near the peak of $\frac{1}{s} \frac{\left|\vec{P}^{\prime}\right|}{|\vec{P}|}$. Therefore, the energy where the maximum of cross section occurs is mainly determined by the maximum of $\frac{1}{s} \frac{\left|\vec{P}^{\prime}\right|}{|\vec{P}|}$.

While the center-of-mass energy $\sqrt{s}$ exceeds the values shown in Table 2 , cross sections decrease. It is interesting to evaluate the intrinsic momenta of quark-antiquark relative motion at high energies, for example, where the cross section is one fourth of the maximum cross section. The average of the quark-antiquark relative momenta of the four mesons in an endothermic reaction is listed in Table 3. If the total mass of final mesons is large, the average relative momentum is large. The differences of the average relative momenta of the reactions in Table 3 are not large. The average relative momenta stay in the rapid falling region of the relative-motion wave functions.

We study meson-meson scattering $A+B \rightarrow C+D$ in the Born approximation. The interaction that affects the scattering is between $A$ constituents and $B$ constituents or between $C$ and $D$. The former must be followed by constituent rearrangement, i.e. the scattering is described by the "prior" diagrams of Fig. 1. The latter must be preceded by constituent rearrangement, i.e. the scattering is described by the "post" diagrams of Fig. 1. In principle, we may use either the "prior" diagrams or the "post" diagrams to study the process $A+B \rightarrow C+D$. But practical calculations may infer the post-prior discrepancy. In this case the prior form is first used to get a result and next the post form is used to get another result. The average of the two independent results is taken to describe the process 
Table 3: Average quark-antiquark relative momentum.

\begin{tabular}{ccc}
\hline reaction & $\sqrt{s}(\mathrm{GeV})$ & average relative momentum $(\mathrm{GeV})$ \\
\hline$I=2 \pi \pi \rightarrow \rho \rho$ & 2.347 & 0.491 \\
$I=2 \pi \rho \rightarrow \rho \rho$ & 2.363 & 0.473 \\
$I=1 K K \rightarrow K^{*} K^{*}$ & 2.703 & 0.580 \\
$I=1 K K^{*} \rightarrow K^{*} K^{*}$ & 2.712 & 0.570 \\
$I=\frac{3}{2} \pi K \rightarrow \rho K^{*}$ & 2.411 & 0.545 \\
$I=\frac{3}{2} \pi K^{*} \rightarrow \rho K^{*}$ & 2.513 & 0.550 \\
$I=\frac{3}{2} \rho K \rightarrow \rho K^{*}$ & 2.507 & 0.553 \\
$I=\frac{3}{2} \pi K^{*} \rightarrow \rho K$ & 1.796 & 0.445 \\
\hline
\end{tabular}

$A+B \rightarrow C+D$. The average in fact avoids double counting in the use of the prior form and the post form, and reconcile the two independent results. For elastic scatterings where the post-prior discrepancy is negligible, we can only employ the post form in comparison to antisymmetrizing the initial state in the resonating group method [19] and generator coordinate method [40]. For inelastic scattering antisymmetrizing the final state in the resonating group method and generator coordinate method is also necessary since the interaction exchange kernel resulted from antisymmetrizing the final state probably differs from the one resulted from antisymmetrizing the initial state. The post and prior forms are related to antisymmetrizing the initial and final states, respectively.

The relationship of the Born series for elastic scattering to the resonating group method or the generator coordinate method [40] was detailed by Barnes and Swanson [16,41]. Born-order $T$ matrix is the interaction exchange kernel in the first term of the iterative procedure of the resonating group method. The exchange kernel for the pure quark exchange with no gluon exchange is proportional to the normalization kernel. It is damped when the cluster-cluster distance approaches infinity and hence does not contribute to scattering $[41,42]$. The Born approximation does not provide a term corresponding to the exchange kernel for the pure quark exchange with no gluon exchange.

Distinct roles of the inelastic scatterings of ground-state mesons can be identified. For instance, $\pi$ absorption reactions are $\pi \pi \rightarrow \rho \rho, \pi \rho \rightarrow \rho \rho, \pi K \rightarrow \rho K^{*}, \pi K^{*} \rightarrow$ $\rho K^{*}, \pi K^{*} \rightarrow \rho K, \pi \phi \rightarrow K K^{*}$ (or $\left.K^{*} K\right)$ and $\pi \phi \rightarrow K^{*} K^{*} ; K^{*}$ creation reactions are $K K \rightarrow K^{*} K^{*}, K K^{*} \rightarrow K^{*} K^{*}, \pi K \rightarrow \rho K^{*}, \rho K \rightarrow \rho K^{*}, \rho K \rightarrow \pi K^{*}, \pi \phi \rightarrow K K^{*}$ (or $\left.K^{*} K\right), \pi \phi \rightarrow K^{*} K^{*}, \rho \phi \rightarrow K K^{*}$ (or $K^{*} K$ ) and $\rho \phi \rightarrow K^{*} K^{*}$. Our calculations give the $\sqrt{s}$-dependent cross sections for the $\phi$ absorption reactions including the notable $I=1 \rho \phi \rightarrow K^{*} K^{*}$ and the average cross sections that depend on temperature of hadronic 
matter. Every endothermic reaction in Subsections 4.2, 4.3 and 4.4 has a maximum cross section larger than the cross section for its corresponding inverse exothermic reaction at the same center-of-mass energy except the reaction of $I=3 / 2 \pi K^{*} \rightarrow \rho K$. Subsequently, $K^{*}$ and $\rho$ from the creation reactions with the appreciable cross sections are expected to compensate for the loss due to decay in hadronic matter and to make $K^{*}$ and $\rho$ observable in $\mathrm{Au}+\mathrm{Au}$ collisions.

\section{Summary}

We have studied meson-meson inelastic scatterings with quark-antiquark relativemotion wave functions that are solutions of the Schrödinger equation with the BuchmüllerTye potential. The inelastic scatterings are governed by the quark-interchange mechanism that gives rise to the prior form and the post form. The post-prior discrepancy is completely negligible in the S-wave elastic phase shifts for $I=2 \pi \pi$ and $I=3 / 2 K \pi$ scattering in spite of that the physical meson masses and flavor-symmetry breaking are employed throughout this work. The two forms do produce different cross sections of which the average is taken as the unpolarized cross section. It is found that the reaction of $I=3 / 2$ $\pi K^{*} \rightarrow \rho K$ is most important among the endothermic nonresonant reactions.

If the difference between $\sqrt{s}$ and threshold energy is the same for the reactions of $I=2$ $\pi \pi \leftrightarrow \rho \rho, I=2 \pi \rho \leftrightarrow \rho \rho, I=1 K K \leftrightarrow K^{*} K^{*}$ and $I=1 K K^{*} \leftrightarrow K^{*} K^{*}$, at this $\sqrt{s}$ the cross sections for $I=1 K K \leftrightarrow K^{*} K^{*}\left(I=1 K K^{*} \leftrightarrow K^{*} K^{*}\right)$ are larger than the cross sections for $I=2 \pi \pi \leftrightarrow \rho \rho(I=2 \pi \rho \leftrightarrow \rho \rho)$. This means that mesonic interactions in matter consisting of only $K$ and $K^{*}$ can be stronger than mesonic interactions in matter consisting of only $\pi$ and $\rho$.

\section{Acknowledgements}

We thank Y.-G. Ma and L.-W. Chen for helpful discussions. After the manuscript was accepted for publication, we got from B.S. Zou an interesting study of $\pi \pi$ elastic scattering which was published in Nucl. Phys. A735 (2004) 111. This work was supported by National Natural Science Foundation of China under Grant No. 10675079. 


\section{References}

[1] S.S. Adler, et al., PHENIX Collaboration, Phys. Rev. C 69 (2004) 034909.

[2] I. Arsene, et al., BRAHMS Collaboration, Nucl. Phys. A 757 (2005) 1.

[3] B.B. Back, et al., PHOBOS Collaboration, Nucl. Phys. A 757 (2005) 28.

[4] J. Adams, et al., STAR Collaboration, Nucl. Phys. A 757 (2005) 102.

[5] K. Adcox, et al., PHENIX Collaboration, Nucl. Phys. A 757 (2005) 184.

[6] J. Adams, et al., STAR Collaboration, Phys. Rev. Lett. 92 (2004) 092301.

[7] I.G. Bearden, et al., BRAHMS Collaboration, Phys. Rev. Lett. 90 (2003) 102301;

I.G. Bearden, et al., BRAHMS Collaboration, Phys. Rev. Lett. 94 (2005) 162301.

[8] J. Bijnens, G. Colangelo, G. Ecker, J. Gasser, M.E. Sainio, Nucl. Phys. B 508 (1997) 263 ;

G. Colangelo, J. Gasser, H. Leutwyler, Nucl. Phys. B 603 (2001) 125, and references therein.

[9] J.A. Oller, E. Oset, nucl-th/9601031, and references therein.

[10] E. Colton, et al., Phys. Rev. D 3 (1971) 2028.

[11] N.B. Durusoy, et al., Phys. Lett. B 45 (1973) 517.

[12] W. Hoogland, et al., Nucl. Phys. B 126 (1977) 109.

[13] M.J. Losty, et al., Nucl. Phys. B 69 (1974) 185.

[14] B. Jongejans, et al., Nucl. Phys. B 67 (1973) 381.

[15] P. Estabrooks, et al., Nucl. Phys. B 133 (1978) 490.

[16] T. Barnes, E.S. Swanson, Phys. Rev. D 46 (1992) 131;

E.S. Swanson, Ann. Phys. 220 (1992) 73.

[17] T. Barnes, E.S. Swanson, J. Weinstein, Phys. Rev. D 46 (1992) 4868.

[18] J. Weinstein, N. Isgur, Phys. Rev. D 43 (1991) 95.

[19] G.-Q. Zhao, X.-G. Jing, J.-C. Su, Phys. Rev. D 58 (1998) 117503.

[20] C.-Y. Wong, E.S. Swanson, T. Barnes, Phys. Rev. C 65 (2001) 014903.

[21] T. Barnes, E.S. Swanson, C.-Y. Wong, X.-M. Xu, Phys. Rev. C 68 (2003) 014903.

[22] Y.G. Ma, J. Phys. G 32 (2006) S373.

[23] J.H. Chen, et al., Phys. Rev. C 74 (2006) 064902.

[24] L.-W. Chen, C.M. Ko, Phys. Rev. C 73 (2006) 044903. 
[25] E. Yamamoto (for the STAR Collaboration), Nucl. Phys. A 715 (2003) 466c;

J. Adams, et al., STAR Collaboration, Phys. Lett. B 612 (2005) 181;

S.S. Adler, et al., PHENIX Collaboration, Phys. Rev. C 72 (2005) 014903;

D. Pal (for the PHENIX Collaboration), Nucl. Phys. A 774 (2006) 489;

M. Oldenburg (for the STAR Collaboration), Nucl. Phys. A 774 (2006) 507.

[26] A. Shor, Phys. Rev. Lett. 54 (1985) 1122.

[27] S.C. Johnson, B.V. Jacak, A. Dress, Eur. Phys. J. C 18 (2001) 645.

[28] L. Bravina, et al., Nucl. Phys. A 715 (2003) 665c.

[29] P.Z. Bi, J. Rafelski, Phys. Lett. B 262 (1991) 485.

[30] C.M. Ko, D. Seibert, Phys. Rev. C 49 (1994) 2198;

S. Pal, C.M. Ko, Z.-W. Lin, Nucl. Phys. A 707 (2002) 525.

[31] K. Haglin, Nucl. Phys. A 584 (1995) 719;

W. Smith, K.L. Haglin, Phys. Rev. C 57 (1998) 1449.

[32] L. Alvarez-Ruso, V. Koch, Phys. Rev. C 65 (2002) 054901.

[33] W. Buchmüller, S.-H.H. Tye, Phys. Rev. D 24 (1981) 132.

[34] X.-M. Xu, Nucl. Phys. A 697 (2002) 825.

[35] J.D. Bjorken, S.D. Drell, Relativistic Quantum Mechanics, (McGraw-Hill, New York, 1964).

[36] T. Barnes, N. Black, E.S. Swanson, Phys. Rev. C 63 (2001) 025204.

[37] C.-Y. Wong, H.W. Crater, Phys. Rev. C 63 (2001) 044907.

[38] N.F. Mott, H.S.W. Massey, The Theory of Atomic Collisions (Clarendon, Oxford, 1965).

[39] S.V. Greene (for the PHENIX Collaboration), Nucl. Phys. A 774 (2006) 93.

[40] I. Bender, H.G. Dosch, H.J. Pirner, H.G. Kruse, Nucl. Phys. A 414 (1984) 359.

[41] T. Barnes, E.S. Swanson, Phys. Rev. C 49 (1994) 1166.

[42] M. Oka, K. Yazaki, Prog. Theor. Phys. 66 (1981) 556, 572. 


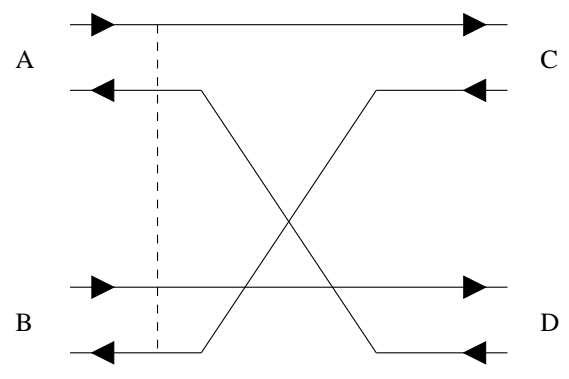

A

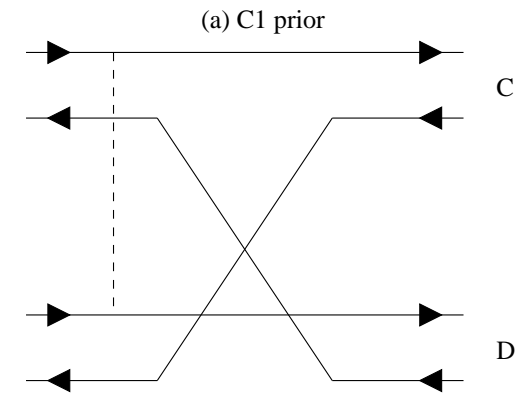

(c) T1 prior

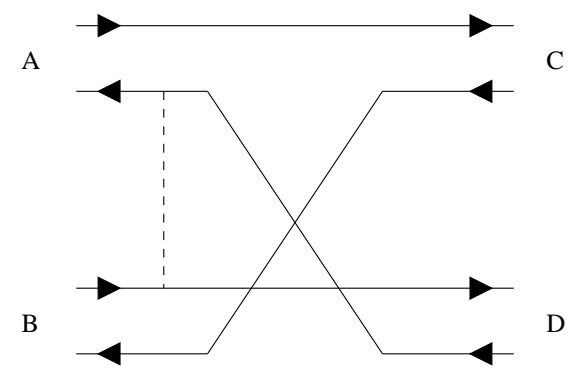

A

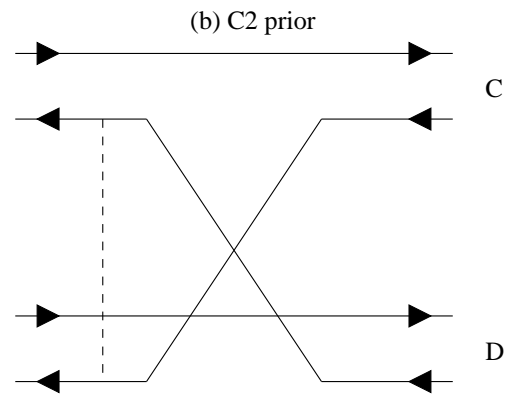

(d) T2 prior

Figure 1: "Prior" diagrams for the reaction $A+B \longrightarrow C+D$. Solid (dashed) lines represent quarks or antiquarks (gluons).

A
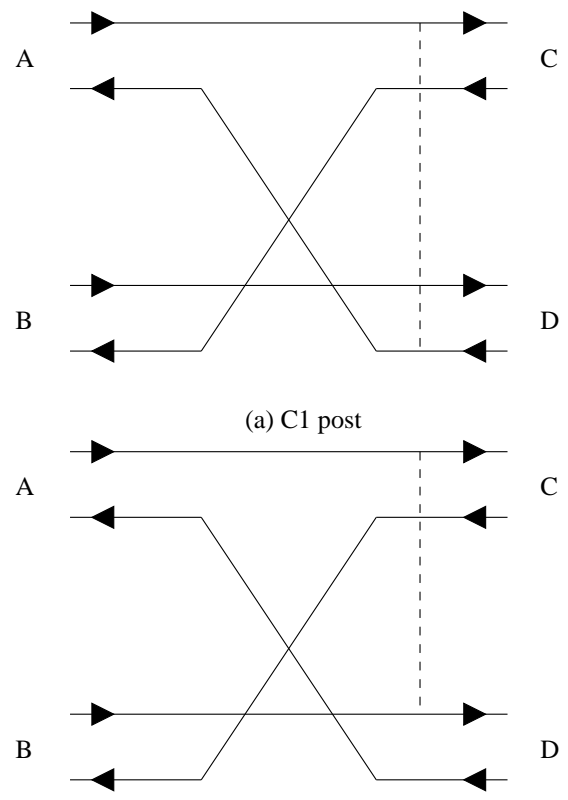

(c) T1 post
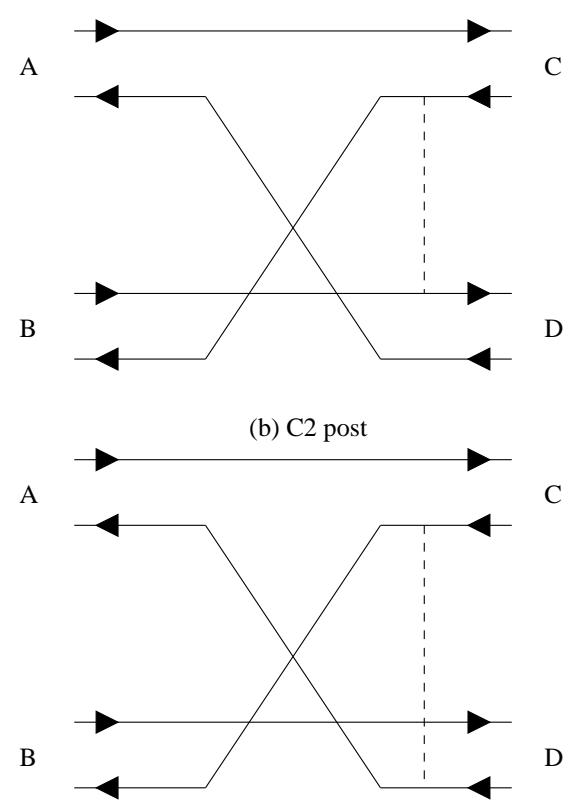

(d) $\mathrm{T} 2$ post

Figure 2: "Post" diagrams. Solid (dashed) lines represent quarks or antiquarks (gluons). 


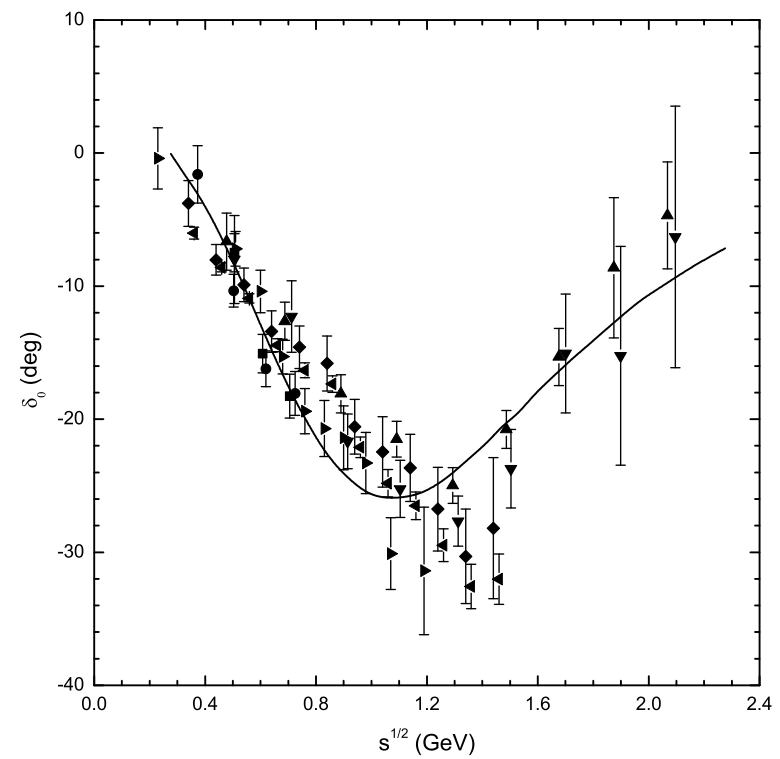

Figure 3: Theoretical S-wave $I=2 \pi \pi$ phase shift (solid curve) compared to experimental data. Squares: Table I of Ref. [10]; circles: Table II of Ref. [10]; triangles up: OPE data of Ref. [11]; triangles down: OPE-DP data of Ref. [11]; diamonds: data of set A of Ref. [12]; triangles left: data of set B of Ref. [12]; triangles right: Ref. [13]. 


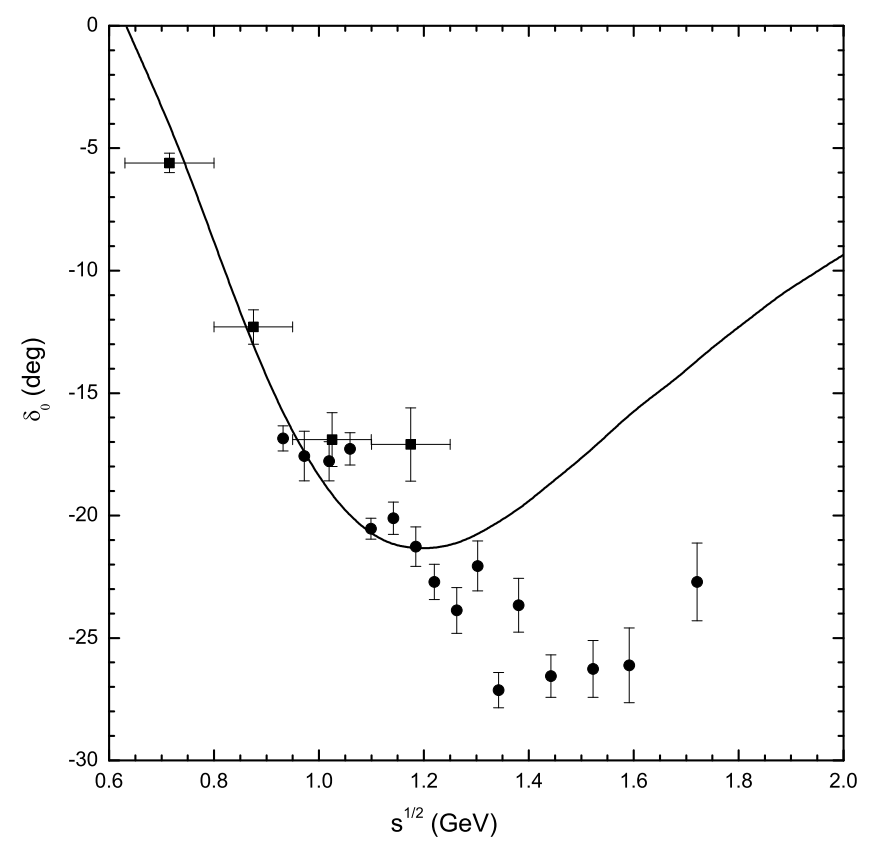

Figure 4: Theoretical S-wave $I=3 / 2 K \pi$ phase shift (solid curve) compared to experimental data. Squares: Ref. [14]; circles: Ref. [15].

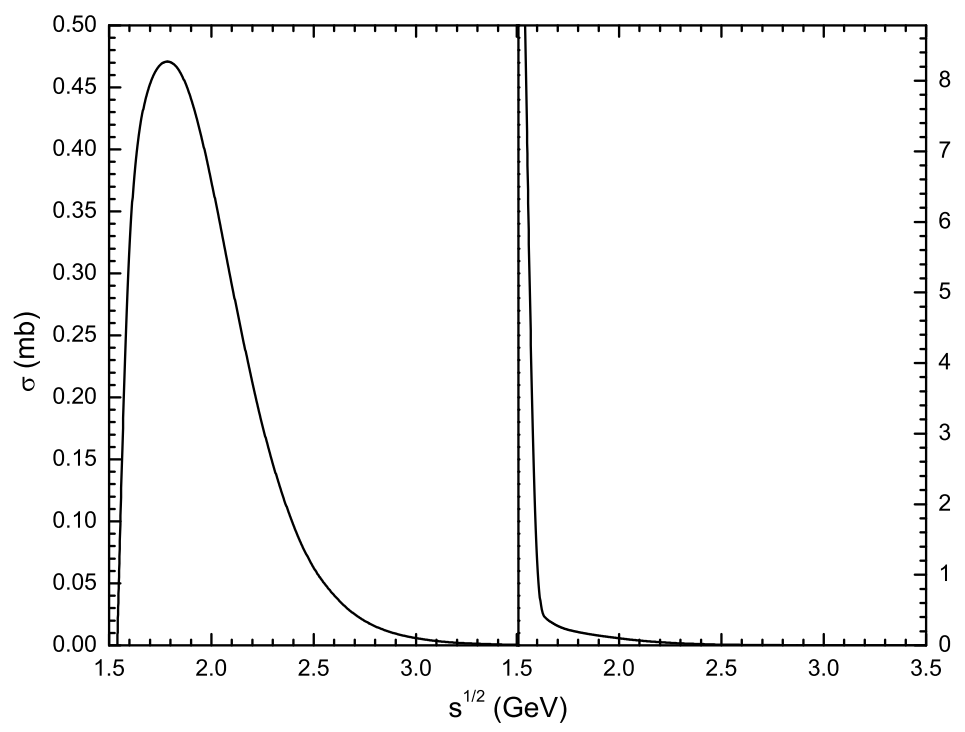

Figure 5: Cross sections for the $I=2 \pi \pi \rightarrow \rho \rho$ in the left panel and for the $I=2 \rho \rho \rightarrow \pi \pi$ in the right panel. 


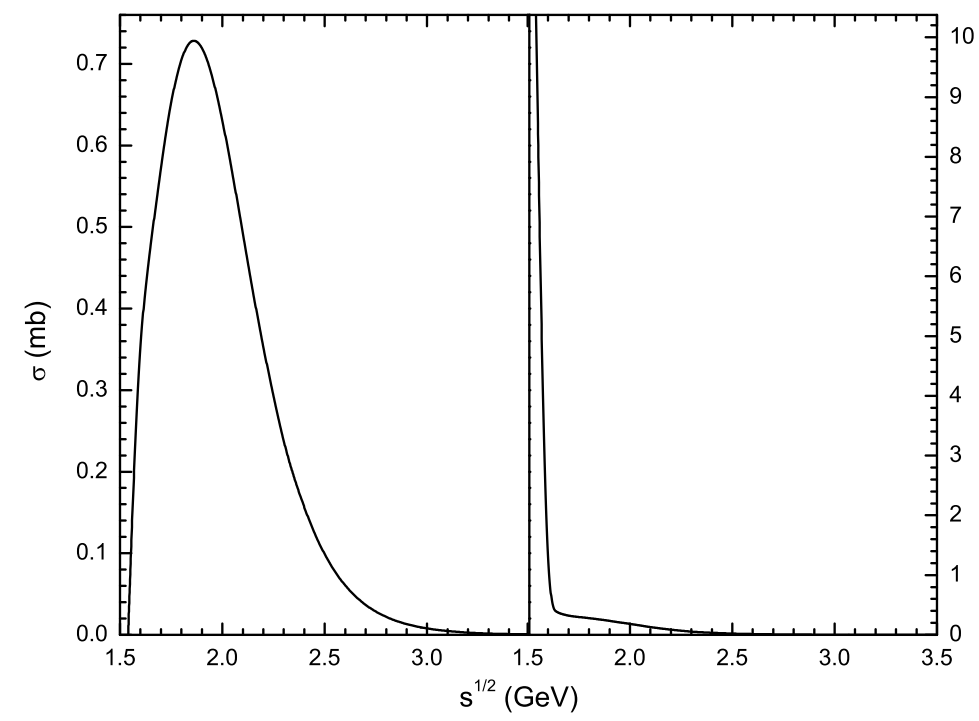

Figure 6: Cross sections for the $I=2 \pi \rho \rightarrow \rho \rho$ in the left panel and for the $I=2 \rho \rho \rightarrow \pi \rho$ in the right panel.

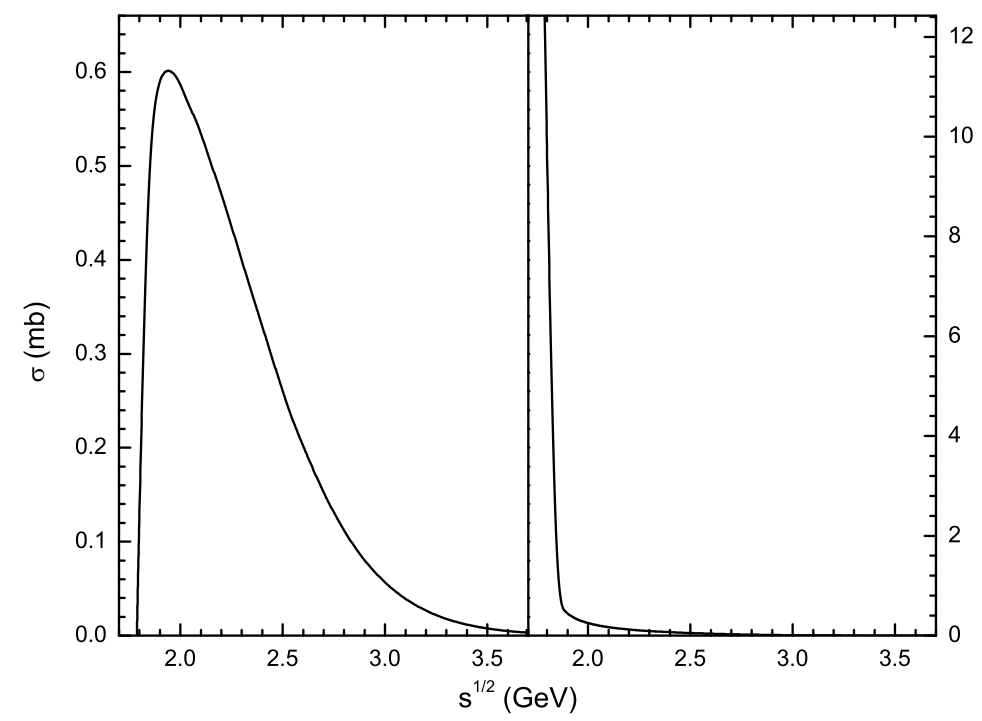

Figure 7: Cross sections for the $I=1 K K \rightarrow K^{*} K^{*}$ in the left panel and for the $I=1 K^{*} K^{*} \rightarrow K K$ in the right panel. 


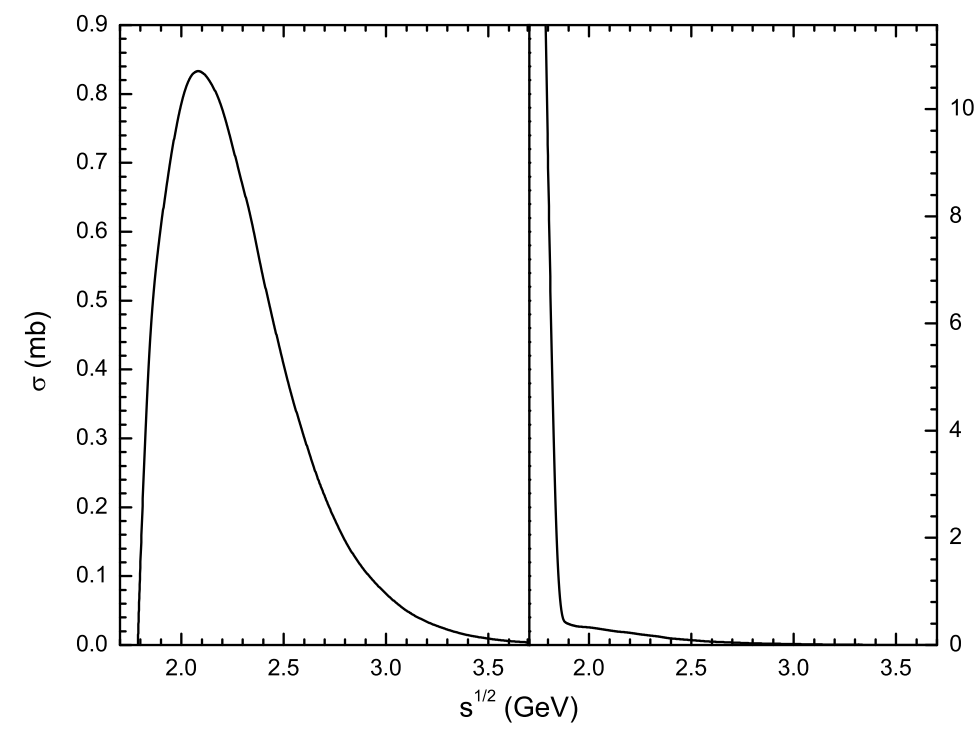

Figure 8: Cross sections for the $I=1 K K^{*} \rightarrow K^{*} K^{*}$ in the left panel and for the $I=1 K^{*} K^{*} \rightarrow K K^{*}$ in the right panel.

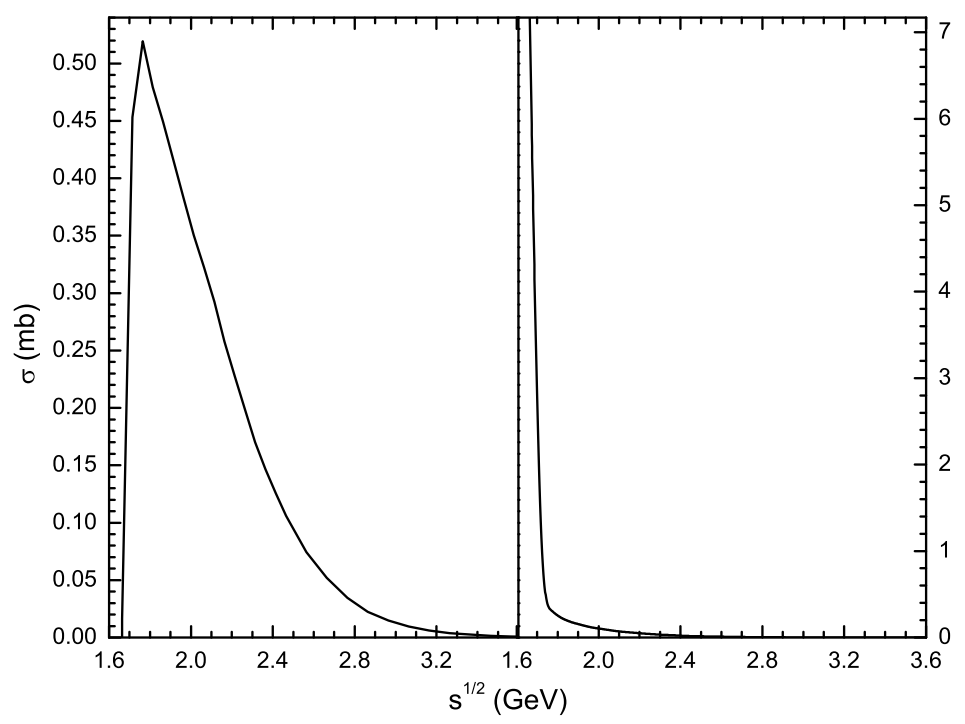

Figure 9: Cross sections for the $I=3 / 2 \pi K \rightarrow \rho K^{*}$ in the left panel and for the $I=3 / 2 \rho K^{*} \rightarrow \pi K$ in the right panel. 


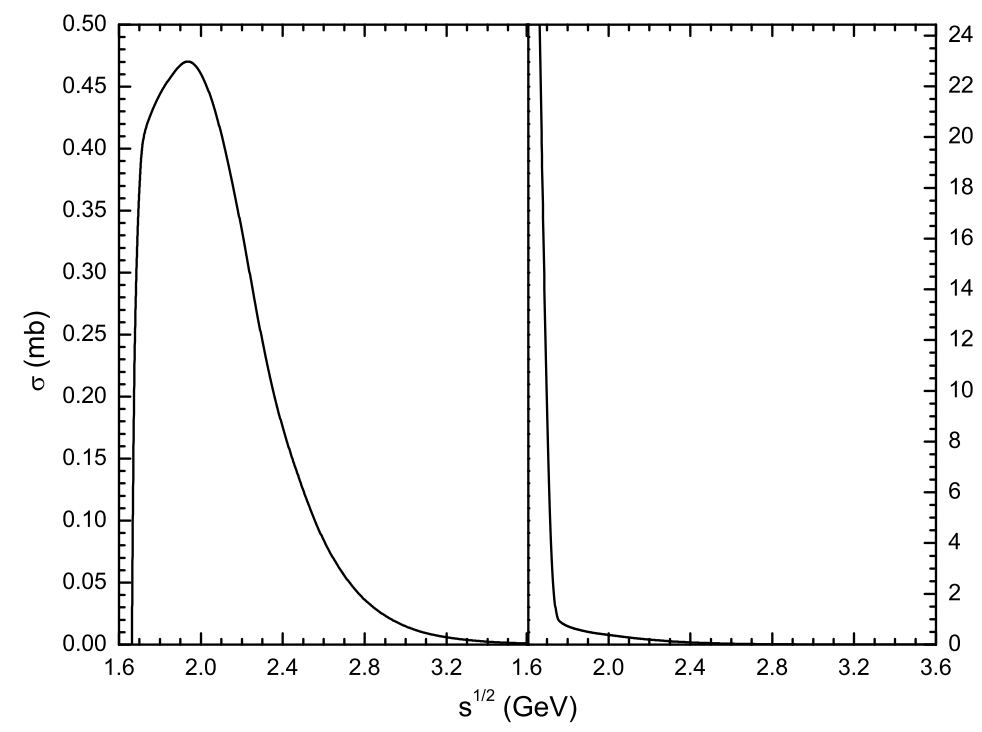

Figure 10: Cross sections for the $I=3 / 2 \pi K^{*} \rightarrow \rho K^{*}$ in the left panel and for the $I=3 / 2 \rho K^{*} \rightarrow \pi K^{*}$ in the right panel.

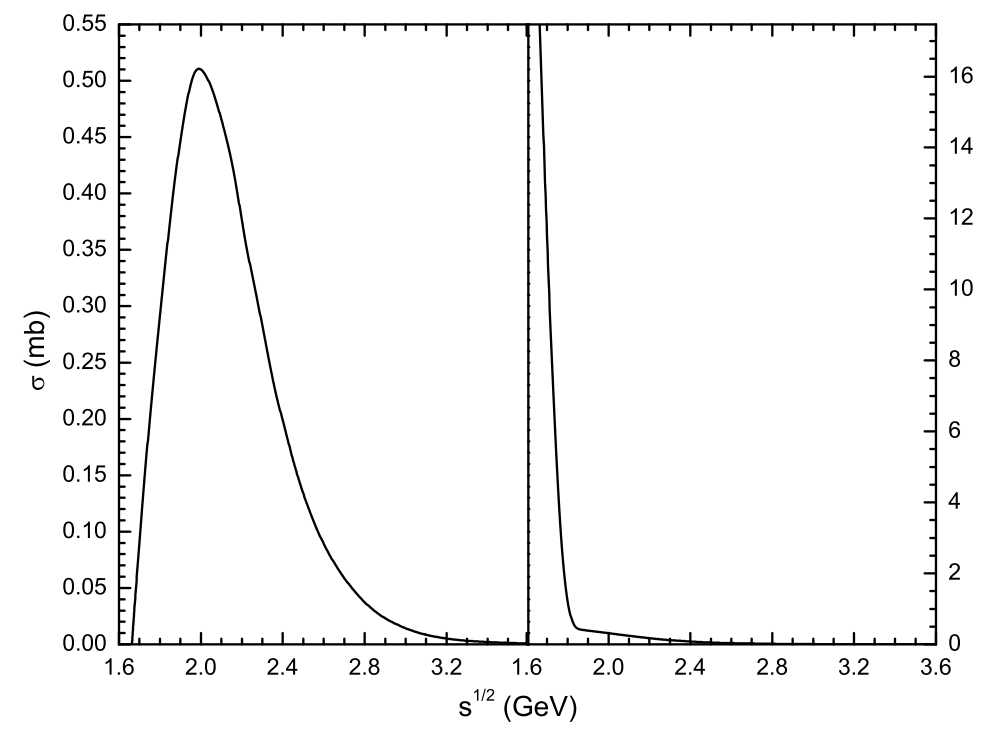

Figure 11: Cross sections for the $I=3 / 2 \rho K \rightarrow \rho K^{*}$ in the left panel and for the $I=3 / 2 \rho K^{*} \rightarrow \rho K$ in the right panel. 


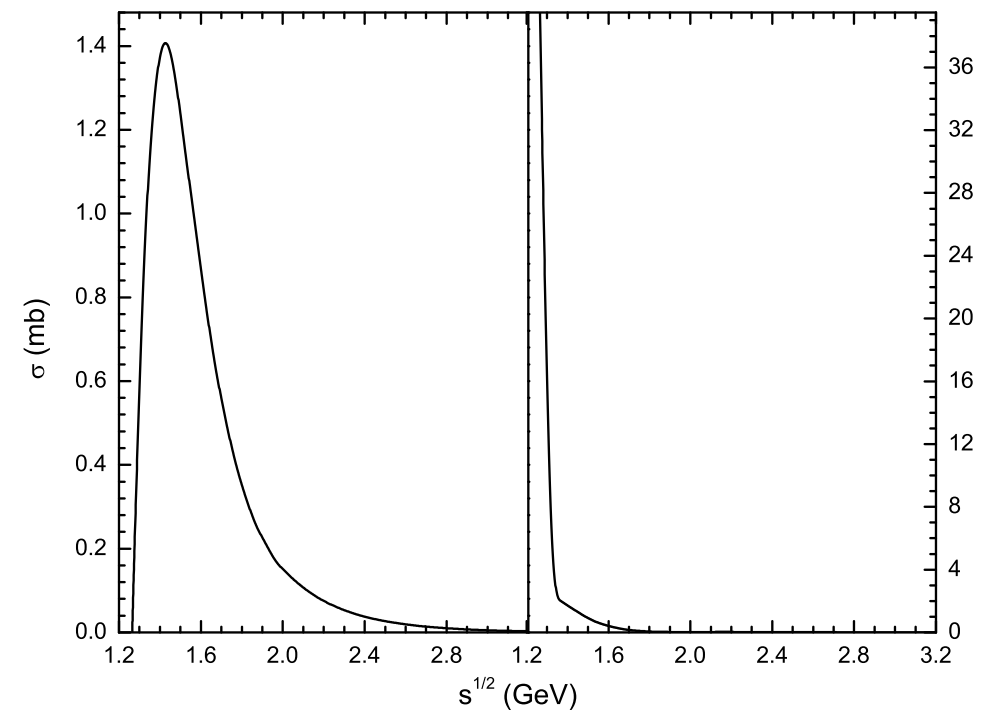

Figure 12: Cross sections for the $I=3 / 2 \pi K^{*} \rightarrow \rho K$ in the left panel and for the $I=3 / 2 \rho K \rightarrow \pi K^{*}$ in the right panel.

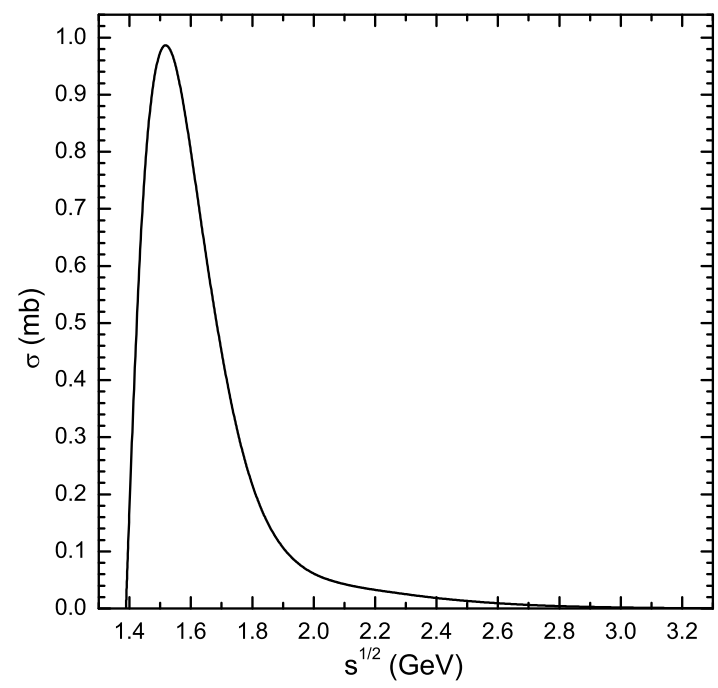

Figure 13: Cross section for the $I=1 \pi \phi \rightarrow K K^{*}\left(\right.$ or $\left.K^{*} K\right)$. 


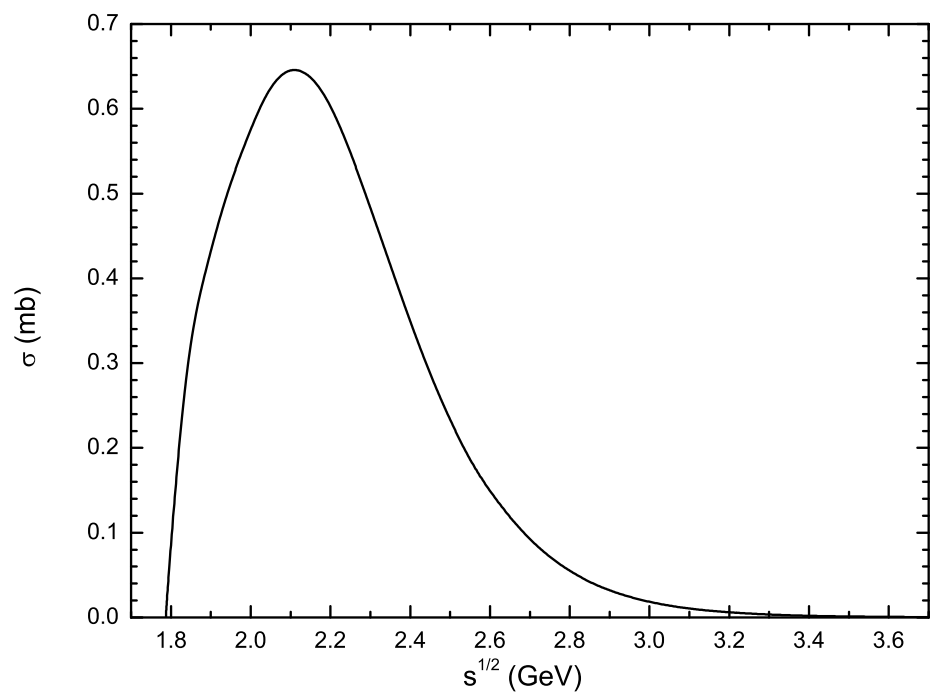

Figure 14: Cross section for the $I=1 \pi \phi \rightarrow K^{*} K^{*}$.

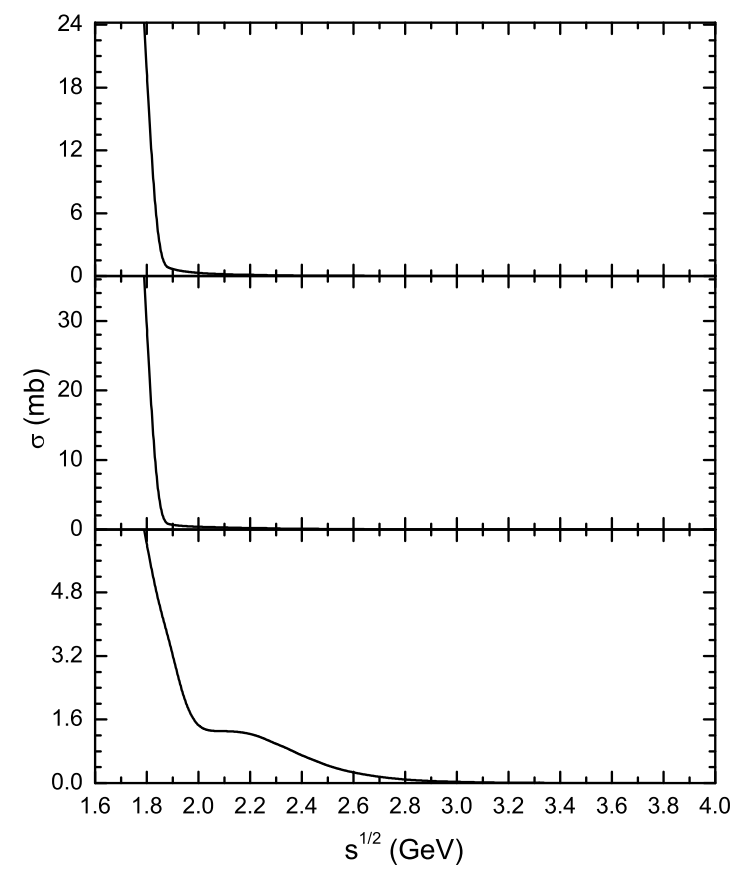

Figure 15: Solid line in the upper (middle, lower) panel shows cross section for the $I=1 \rho \phi \rightarrow K K\left(I=1 \rho \phi \rightarrow K K^{*}\left(\right.\right.$ or $\left.\left.K^{*} K\right), I=1 \rho \phi \rightarrow K^{*} K^{*}\right)$. 